\title{
A Survey Describing Beyond Si Transistors and Exploring Their Implications for Future Processors
}

\author{
HEEWOO KIM, APORVA AMARNATH, JAVAD BAGHERZADEH, NISHIL TALATI, \\ and RONALD G. DRESLINSKI, University of Michigan, Ann Arbor
}

\begin{abstract}
The advancement of Silicon CMOS technology has led information technology innovation for decades. However, scaling transistors down according to Moore's law is almost reaching its limitations. To improve system performance, cost, and energy efficiency, vertical-optimization in multiple layers of the computing stack is required. Technological awareness in terms of devices and circuits could enable informed system-level decisions. For example, graphene is a promising material for extremely scaled high-speed transistors because of its remarkably high mobility, but it can not be used in integrated circuits as a result of the high leakage current from its zero bandgap. In this article, we discuss the fundamental physics of transistors and their ramifications on system design to assist device-level technology consideration during system design. Additionally, various emerging devices and their utilization on a vertically-optimized computing stack are introduced. This article serves as a survey of emerging device technologies that may be relevant in these areas, with an emphasis on making the descriptions approachable by system and software designers to understand the potential solutions. A basic vocabulary will be built to understand how to digest technical content, followed by a survey of devices, and finally a discussion of the implications for future processing systems.
\end{abstract}

CCS Concepts: • General and reference $\rightarrow$ Surveys and overviews; $\bullet$ Hardware $\rightarrow$ 3D integrated circuits; Transistors; Carbon based electronics; Tunneling devices; • Computer systems organization $\rightarrow$ Heterogeneous (hybrid) systems;

Additional Key Words and Phrases: Beyond Moore, emerging devices, system design, III-V, carbon-based devices, Ge nanowire, TFET, NCFET, $\mathrm{SiC}, \mathrm{MoS}_{2}$, emerging non-volatile memory

\section{ACM Reference format:}

Heewoo Kim, Aporva Amarnath, Javad Bagherzadeh, Nishil Talati, and Ronald G. Dreslinski. 2021. A Survey Describing Beyond Si Transistors and Exploring Their Implications for Future Processors. f. Emerg. Technol. Comput. Syst. 17, 3, Article 27 (June 2021), 44 pages.

https://doi.org/10.1145/3453143

\section{INTRODUCTION}

The advances in Silicon-based semiconductor and microprocessor technology in recent decades have changed the world we live in significantly. The improvements in computing power, storage capacity, and communication have led us to the world of mobile devices and world wide web, and are taking us to the era of the Internet of Things (IoT), cloud computing, artificial intelligence,

\footnotetext{
Authors' address: H. Kim, A. Amarnath, J. Bagherzadeh, N. Talati, and R. G. Dreslinski, University of Michigan, Ann Arbor, 2260 Hayward Street, Ann Arbor, Michigan, 48109-2121; emails: \{heewoo, aporvaa, javadb, talatin, rdreslin\}@umich.edu. Permission to make digital or hard copies of all or part of this work for personal or classroom use is granted without fee provided that copies are not made or distributed for profit or commercial advantage and that copies bear this notice and the full citation on the first page. Copyrights for components of this work owned by others than ACM must be honored. Abstracting with credit is permitted. To copy otherwise, or republish, to post on servers or to redistribute to lists, requires prior specific permission and/or a fee. Request permissions from permissions@acm.org.

(C) 2021 Association for Computing Machinery.

1550-4832/2021/06-ART27 \$15.00

https://doi.org/10.1145/3453143
}

ACM Journal on Emerging Technologies in Computing Systems, Vol. 17, No. 3, Article 27. Pub. date: June 2021. 
autonomous vehicle, and so on. In this new world, data is the key element as mentioned in the following phrases: "Data is the new oil" [67], "Data is defining the future of our industry" [134]. The total amount of global data is exploding as the $2.8 \mathrm{ZB}$ in $2012\left(1 \mathrm{ZB}=10^{9} \mathrm{~TB}\right)$ [52], became $32 \mathrm{ZB}$ in 2018, and is expected to reach $175 \mathrm{ZB}$ in 2025 [129]. Consequently, collecting, storing, and processing data will become more and more important and semiconductor technology is expected to contribute to this outburst of data as follows:

- Data collection and Transporting: Sensor networks, embedded systems, and communication platforms $(5 \mathrm{G})$ are the key technologies for IoT and data mining. Novel device technologies (high-temperature or flexible devices) can contribute to collecting data from wider and more omnipresent environments. While low power devices can contribute to configure efficient and long battery life hardware systems.

- Data storage: Servers and cloud systems require large capacity of data storage and ultra high-speed accessibility. High density and low leakage power devices with short latency are required for configuring the memory in these systems. New memories such as spin-transfer-torque magnetoresistive random-access memory (STT-MRAM), phase change random-access memory (PCRAM), resistive random-access memory (RRAM), ferromagnetic RAM (FeRAM), and ferroelectric field-effect transistor (FeFET) are also being investigated for this application.

- Data processing: There is a need for processing data fast and concurrently. High computing power with low energy consumption and large bandwidth are required to meet the growing demands in AI, deep learning, and machine learning. Highly integrated low power devices can play a great role in meeting the needs in data processing with application specific integrated circuits (ASICs) and domain specific computing on the hardware side.

The main advantage of utilizing advanced device technologies is that it provides free lunch on the circuit and architecture level. By using these advanced device technologies on the same design and structure, we can increase the speed without a power tradeoff, or decrease power without a penalty in speed. Hence, development of hardware and software technologies has been supported by the advancement of Si CMOS technology for the past several decades. However, the advancement of Si CMOS devices is reaching its limit, and we need to find new semiconductor devices that can replace Si CMOS. Despite the numerous studies and introduction of several new device technologies, there is no dominant candidate that could replace current Si CMOS for the full application spaces $[10,14,26,70]$. Instead, we can utilize emerging devices for specific purposes and applications as they offer unique features and properties, similar to the rise of ASIC and domainspecific processors due to the CPU performance saturation.

Emerging devices can contribute to the growing demand in computing power by being applied in extreme environments and conditions that Si cannot, or provide higher density and better performance with low power (low energy-delay-delay-product (EDDP)). Over the past decades, the semiconductor industry had to deal with several challenges and limitations such as gate leakage current, off-state current, and gate controllability, which has been resolved using innovative semiconductor technologies, such as strained Si CMOS technology from the $90 \mathrm{~nm}$ node, high$\kappa$ metal gate transistors from the $45 \mathrm{~nm}$ node, and FinFETs from the $22 \mathrm{~nm}$ node [17]. Similarly, emerging devices can provide the breakthrough to the Si CMOS physical and structural limitations. In addition, co-optimization in device and architecture level can further enhance the performance. However, to date these technologies are rarely discussed beyond the in-depth physics-based papers, which make them hard to access by non-device level experts. To shed more light on how these emerging devices are one of the key components of this new computing era and provide a 


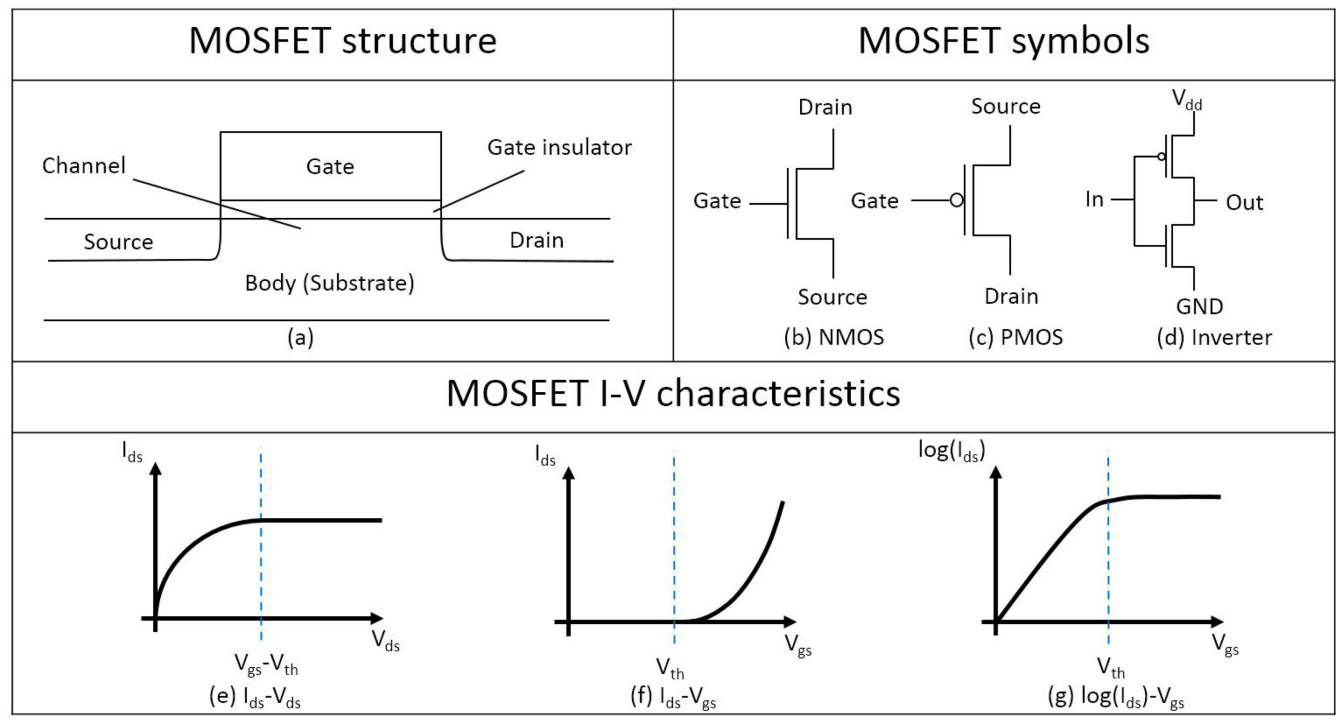

Fig. 1. MOSFET structure and current-voltage graph.

more easily consumable description by non-experts, we will introduce their physics, principles, properties of devices, and how the emerging devices can be applied and affect the system level.

In this article, we will go through the properties and physics of beyond Si transistors, and how those properties can contribute to the hardware improvements for abundant data computing. We will first take a look at the physical properties of device technologies and how these properties impact design trade-offs. We will also describe how the physical properties can affect the EDDP and transistor density. After that, we will introduce several emerging devices with their physical properties, advantages and disadvantages, and the application of these devices at the architecture level.

\section{FUNDAMENTAL PHYSICS OF TRANSISTORS}

In this section, we build up the vocabulary of devices. The intention here is to educate the nondevice experts about the key terminology and functioning of semiconducting devices. This vocabulary will later be used to aid in the understanding of emerging device technologies and their implications. The intended audience for this section is software and system level designers who may understand gate level design, but have little to no familiarity with device characteristics. This section also aids more expert readers in using the same vocabulary.

\subsection{Basic Terminologies and Transistor Structure}

The transistor is a three-terminal electrical switch. Metal-oxide-semiconductor field-effecttransistor (MOSFET) is a commonly used transistor for recent digital integrated circuits. There are two types of MOSFET, which are n-type and p-type. The n-type transistor (see Figure 1(b)) is good for pulling down the logic state from 1 to 0 , and the p-type transistor (see Figure 1(c)) is good for pulling up the logic state from 0 to 1 . A logical inverter is made of the n-type transistor (NMOS, nFET) and the p-type transistor (PMOS, pFET), as shown in Figure 1(d). The terminals of MOSFET are the source, drain, and gate as shown in Figure 1(a). The source supplies charge carriers, which are electrons for n-type MOSFET and holes for p-type MOSFET. The drain collects charge carriers that come from the source. The gate controls the source to drain charge carrier 
flow, which is the transistor current. The channel is the current path that connects the source and drain. The source, drain, and channel are located at the bulk semiconductor, which is called the body. The gate oxide is the insulator that electrically isolates the gate and the channel. To summarize, MOSFET is a three-terminal electrical switch and the gate voltage is used to control whether there is a connection or isolation between the source and the drain.

Impurity doping is the main mechanism in Si MOSFET that makes the semiconductor material conductive. About one in a million $\mathrm{Si}$ atoms is replaced with an impurity atom, which does not disturb the Si lattice structure but it is enough to change the semiconductor's electrical properties. Group V elements such as phosphorus, arsenic, and antimony are used for n-type doping. Group III elements such as boron, aluminum, and indium are used for p-type doping. Group V elements have five valence electrons, which results in one excessive electron in the Si lattice structure, since $\mathrm{Si}$ has four valence electrons. However, group III elements have three valence electrons, which results in one deficient electron in the Si lattice structure, and the electron vacancy behaves like a positive charge.

Pure Si without impurity doping has some level of carrier concentration, which is called the intrinsic carrier concentration $\left(n_{i}\right)$. The intrinsic carrier concentration, which is $1.5 \times 10^{10} \mathrm{~cm}^{-3}$ for $\mathrm{Si}$ at $300 \mathrm{~K}$, comes from the thermal excitation of Si's electrons and holes. This number is significantly lower than the n-type or p-type semiconductor carrier concentration, which is about $10^{16} \sim 10^{19} \mathrm{~cm}^{-3}$.

The drain to source voltage $\left(V_{d s}, V_{d}\right)$ and gate to source voltage $\left(V_{g s}, V_{g}\right)$ are the key parameters of controlling the transistor current $\left(I_{d s}, I_{d}\right)$ (see Figures 1(e) 1(g)). As shown in Figure 1(e), when $V_{d s}$ increases and $V_{g s}$ remains constant, $I_{d s}$ increases quadratically until $V_{d s}=V_{g s}-V_{t h}\left(V_{t h}\right.$ is threshold voltage) and remains constant when $V_{d s}$ goes above this voltage. This means that $I_{d s}$ is independent of $V_{d s}$ and $V_{d s}$ does nothing for the current switching when $V_{d s}$ is above $V_{g s}-V_{t h}$. As shown in Equation (1), this is the case for digital circuits, since $V_{d s}$ is set to $V_{d d}$ and $V_{g s}$ is set to 0 or $V_{d d}$ :

$$
V_{d s}=V_{d d}>V_{d d}-V_{t h} \geq V_{g s}-V_{t h} .
$$

It is $V_{g s}$ that controls the transistor current flow. As shown in Figure 1(f), when $V_{g s}$ increases and $V_{d s}$ remains constant, the transistor is off when $V_{g s}$ is below $V_{t h}$, and is on when $V_{g s}$ becomes higher than $V_{t h}$. In reality, the transistor is not fully turned off when $V_{g s}$ is lower than $V_{t h}$. In the $\log$ scale $I_{d s}-V_{g s}$ graph (see Figure $1(\mathrm{~g})$ ), the transistor still conducts current even when $V_{g s}$ is below $V_{t h}$. This is the reason for static leakage power consumption. To summarize, $V_{g s}$ controls the transistor switching and $V_{d s}$ does nothing for the transistor switching. Furthermore, the transistor is not perfectly turned off when below threshold or even in the off-state, leading to the leakage power consumption.

\subsection{A Single-transistor Level}

This section describes the fundamental physics of a single transistor that will help to understand the emerging device properties and applications. The concepts of current flow mechanisms, mobility, carrier concentration, off-state current, on/off steepness, and bandgap are discussed, and summarized in Table 1.

2.2.1 Current Flow Mechanism. This section discusses two different current flow mechanisms of emerging devices. The first one is thermionic emission modulation, and the second one is bandto-band tunneling. Schottky barrier tunneling mechanism is another type of quantum tunneling mechanism [53, 111], which will be further discussed in Section 5.2.2.

Thermionic Emission Modulation. A transistor is an electrical switch in which the gate voltage controls the source to drain current. The switching mechanism that is widely used for typical 
Table 1. Current Flow Mechanisms and Physical Properties of Transistors






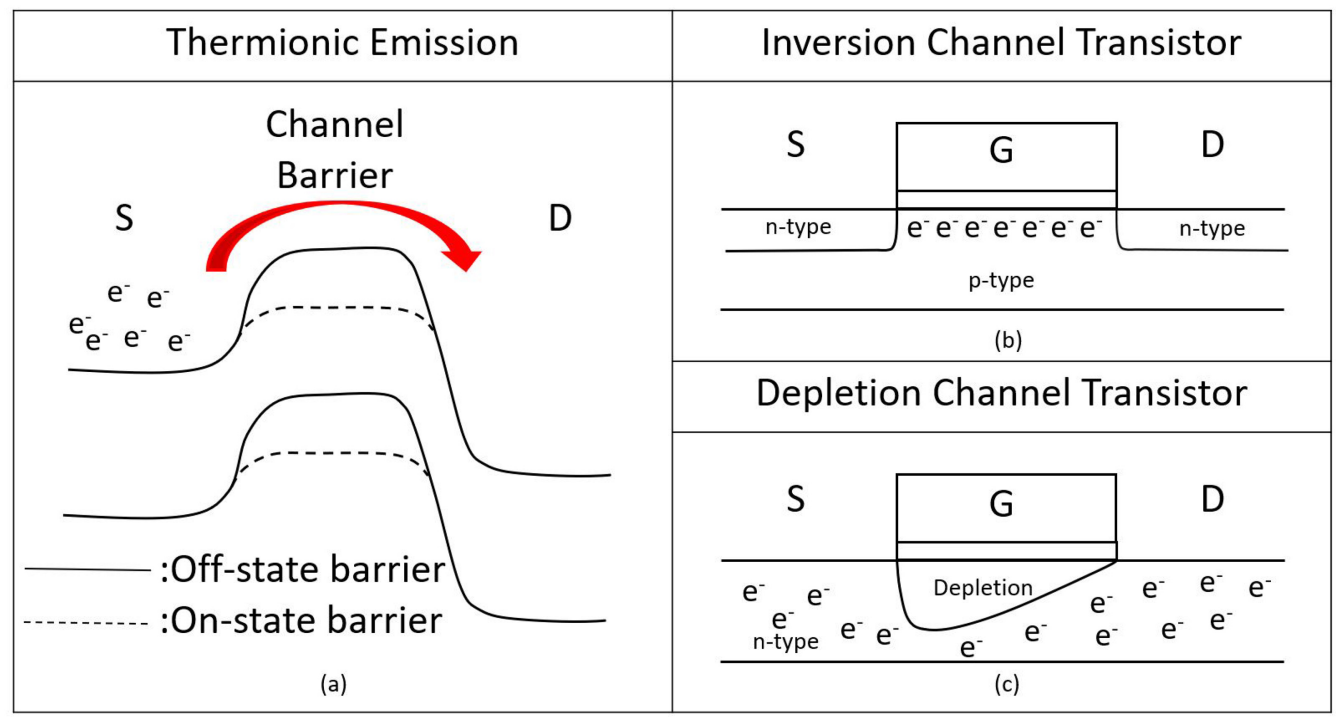

Fig. 2. Thermionic emission, inversion channel transistor and depletion channel transistor that use thermionic emission.

transistors is thermionic emission modulation. Thermionic emission means that when the temperature is not absolute zero $(0 \mathrm{~K})$, electrons that are bound into semiconductor atoms are thermally excited and move free, which makes the electrons behave as free electrons. Thermally emitted charge carriers flow from source to drain when a source to drain voltage is applied. However, the energy barrier in the channel region, which is controlled by the gate voltage, blocks the charge carrier flow. Current flow is controlled by the channel energy barrier height as shown in Figure 2(a). Thermionic emission modulation can be compared to a hill, a bump, and balls. The source is analogous to the upper part of a hill, the drain is analogous to the lower part, the channel energy barrier is analogous to a bump and balls are analogous to electrons. The balls themselves have the kinetic energy, so they wander around the upper part of the hill. The bump makes the balls stay in the upper part of the hill and prevents the balls from falling to the lower part of a hill. However, when the height of the bump becomes lower, some of the balls cross the bump and fall to the lower part of the hill.

There are two types of transistors that are in thermionic emission category. The first one is the inversion channel transistor, and another is the depletion channel transistor (junctionless transistor) [35].

The major structural difference between the inversion channel transistor and depletion channel transistor is that the inversion channel transistor has $n^{+}-p-n^{+}$(source channel drain) structure (see Figure 2(b)), but the depletion channel transistor has $n^{+}-n-n^{+}$structure (see Figure 2(c)). For the inversion channel transistor, when the gate voltage is applied for $\mathrm{n}$-type MOSFET, a two-dimensional electron layer is formed at the gate insulator/p-channel interface, and this charge layer becomes the current conducting path. The two-dimensional electron layer is called an inversion layer, because they are formed at the opposite type (p-type) semiconductor. For the depletion channel transistor, when the gate voltage is applied, the depletion layer widens and blocks the current flow from source to drain in the n-type doped channel. The depletion layer (depletion area) is the electrically insulated region that neither the electron nor the hole exists. 


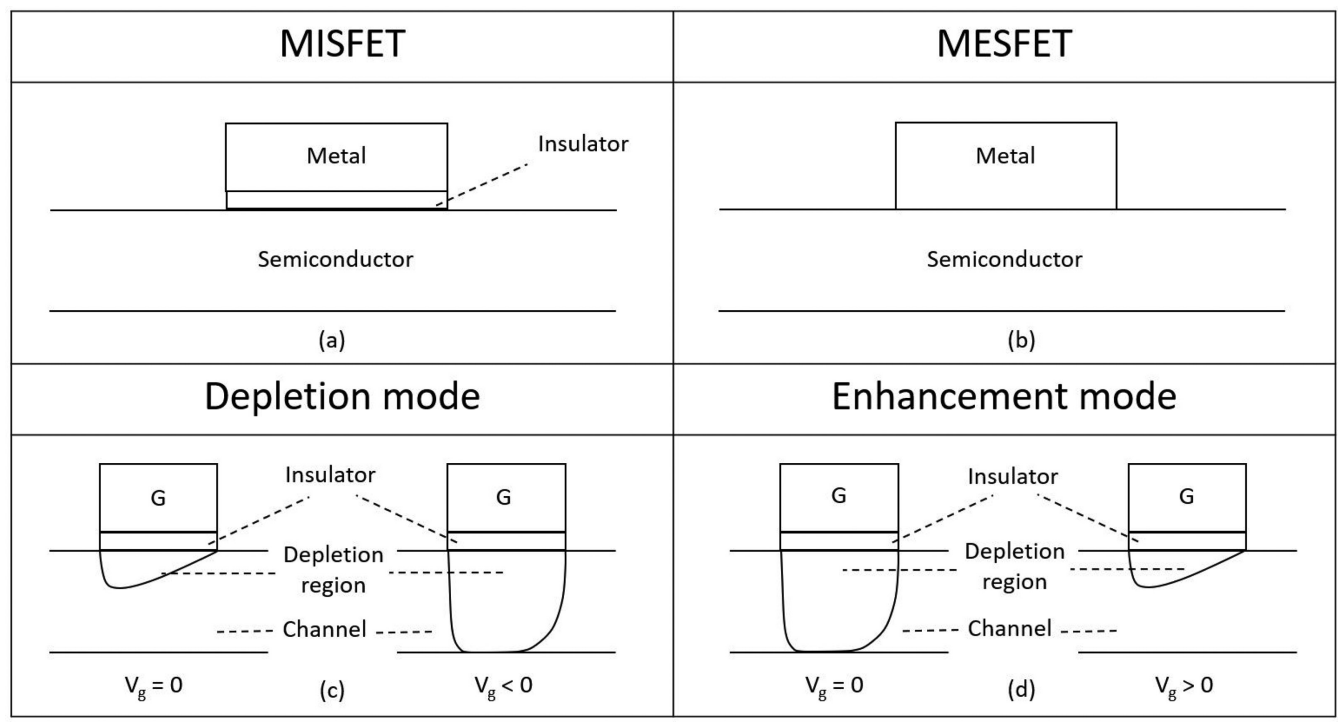

Fig. 3. Depletion channel transistors : MISFET, MESFET, depletion mode, and enhancement mode.

Inversion channel transistors are widely used for current Si CMOS devices. However, depletion channel transistors are widely used for non-Si-based emerging devices [35]. The reason is that depletion channel devices do not require an abrupt $\mathrm{p}-\mathrm{n}$ junction and high-quality dielectricsemiconductor interface, and emerging materials have inferior quality compared to $\mathrm{Si}$ in the aspect of p-type doping and defect concentration.

The existence of the gate dielectric insulator material classifies thermionic emission transistors into metal-semiconductor field-effect transistor (MESFET) and metal-insulator-semiconductor field-effect transistor (MISFET). While the MISFET has a gate insulator between the gate and the channel (see Figure 3(a)), the MESFET does not (see Figure 3(b)), leading to a simpler fabrication process than the MISFET, and hence, MESFETs can be used when there is no suitable gate dielectric insulator. However, this leads to a limitation on the gate voltage range, since there is no gate insulator that electrically isolates the gate and the channel, thus forward-biasing the metalsemiconductor junction inducing a huge gate current. The metal-oxide-semiconductor fieldeffect transistor (MOSFET), which is a subset of the MISFET, uses oxide materials such as silicon oxide or hafnium oxide as a gate insulator. Currently, MOSFET is the most widely used form of transistors, while some emerging devices such as SiC transistors use the MESFET structure [113, 116, 117].

The polarity of the threshold voltage classifies depletion channel transistors into depletion mode and enhancement mode. Depletion mode is when the transistor is turned on at zero gate voltage and is turned off at negative gate voltage, which means that it has a negative threshold voltage (see Figure 3(c)). Enhancement mode is when the transistor is turned off at zero gate voltage and is turned on at positive gate voltage, which means that it has a positive threshold voltage (see Figure 3(d)). Enhancement mode is desirable for digital circuit and processor implementation, since 0 and $V_{d d}$ can represent the logic state 0 and $1\left(0<V_{t h}<V_{d d}\right)$. However, depletion mode transistors require additional voltage level shifting circuits to be used in digital circuits, since both 0 and $V_{d d}$ represent the on-state $\left(V_{t h}<0<V_{d d}\right)$. Instead, depletion mode transistors can be used for analog circuit components such as load resistor, voltage follower, and RF power amplifier [56]. The enhancement mode transistors are harder to fabricate than the depletion mode transistors, 


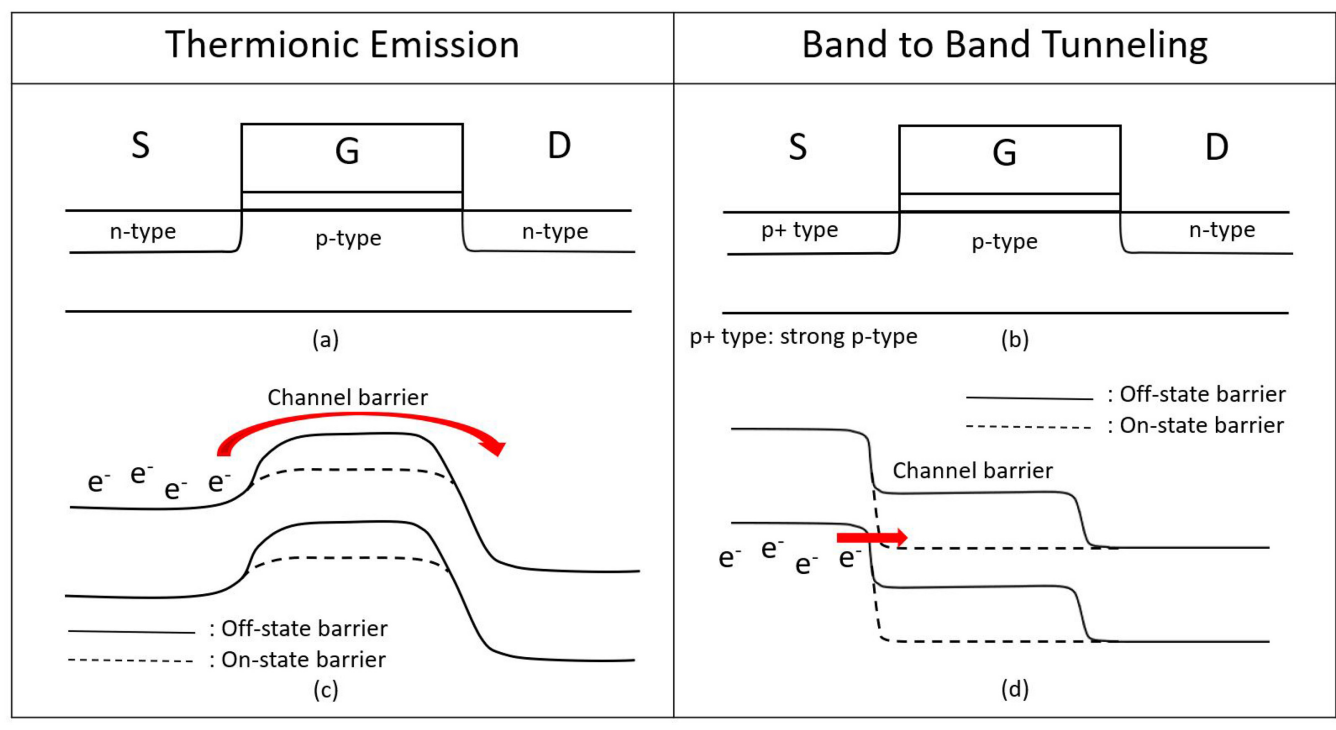

Fig. 4. Thermionic emission and band to band tunneling.

since the enhancement mode transistor's channel thickness should be thin enough to be turned off at zero voltage.

Band-to-band Tunneling. Band-to-band tunneling is the quantum tunneling of electrons and holes through the barrier (see Figure 4(d)). Because of the wave-particle duality, an electron is a particle but also behaves as a wave so it can penetrate a very thin barrier. Band-to-band tunneling can be compared to the sound, two people, and a very high and wide wall. The sound is analogous to the electron, the very high and wide wall is analogous to the channel energy barrier. One person is making a sound at one side of the wall and another person is trying to listen to the sound from the other side of the wall. Since the wall is very high and wide, it blocks the sound propagating to the other side. However, with a thinner wall, another person can hear the sound, because the sound can penetrate the thin wall.

Band-to-band tunneling transistor was proposed to overcome the voltage scaling limitation of the thermionic emission transistor. The structure of the tunneling transistor is similar to the structure of the MOSFET, except that the source region is strongly p-type doped (see Figure 4(b)) instead of n-type doped (see Figure 4(a)). The voltage scaling limitation is due to the increase of off-state current, since the current on/off slope steepness has an upper limitation. The inverse of the ratio of source to drain current $\left(\Delta I_{d s}\right)$ change to gate voltage change $\left(\Delta V_{g s}\right)$, which is called the subthreshold swing $\left(\frac{\Delta V_{g s}}{\log _{10} \Delta I_{d s}}\right)$, cannot be smaller than $60 \mathrm{mV} / \mathrm{dec}$ in thermionic emission transistor. Gate voltage should be changed by at least $0.06 \mathrm{~V}$ to modify the channel current by 10 times. Unlike thermionic emission transistor, which controls the current flow with channel energy barrier height as shown in Figure 4(c), band-to-band tunneling transistor controls the current flow with channel barrier thickness and electron tunneling as shown in Figure 4(d). Since band-to-band tunneling drain current is super-exponential to the gate voltage, subthreshold swings lower than $60 \mathrm{mV} / \mathrm{dec}$ can be achieved [73].

2.2.2 On-state Current: Mobility and Carrier Concentration. A transistor's on-state current $\left(I_{o n}\right)$ is one of the critical determinants for circuit and processor's delay $\tau$, defined as $\tau=\frac{C V}{I_{o n}}$, where $C$ is the load capacitance, $V$ is the supply voltage and $I_{o n}$ is the transistor source to drain on-state 




Fig. 5. Mobility degradation when doping concentration increases for silicon [63].

current. Achieving higher $I_{o n}$ by improving the material quality or by using new transistor materials helps accomplish a smaller latency for the same circuit and architecture design, technology node, and power consumption. The reason is that dynamic power consumption is only related to activity factor $\alpha, C$, and $V\left(P_{d y n}=\alpha C V^{2}\right)$, and $C$ and $V$ are determined by circuit and architecture design, technology node, and device geometry. From $I=J A$ (where $J$ is current density and $A$ is area) and $J=e n \mu E$ (where $e$ is the electron charge, $n$ is the carrier concentration, $\mu$ is the mobility, and $E$ is the electric field), high $I_{o n}$ can be achieved by high mobility $(\mu)$ and high carrier concentration $(n)$. Mobility is defined by how fast the charge carrier (electron, hole) is moving when the external electric field is applied $(v=\mu E$, where $v$ is the drift velocity, $\mu$ is the mobility, and $E$ is the electric field). Carrier concentration is defined by how dense the charge carriers exist in the semiconductor. It is essential to have a high carrier concentration with high mobility for fast processor performance. However, for semiconductors, carrier concentration and mobility are in a trade-off relation. High carrier concentration is achieved by impurity doping, but high impurity doping results in mobility degradation as a result of the increase of collisions between dopant impurities and charge carriers (see Figure 5). There are several emerging devices, such as CNFET (carbon nanotube field-effect transistor) [19, 74], Ge nanowire [25, 159], or InGaAs transistor [142], that achieve high carrier concentration without impurity doping, so they can have a high carrier concentration without mobility degradation.

2.2.3 Off-State Leakage Current and On/Off Steepness (Subthreshold Swing). Power consumption has become a primary constraint for modern processor design [80]. As transistor sizes become smaller and smaller, static power dissipation constitutes a bigger portion among total power consumption $[61,80]$. Static power consumption is especially important for SRAM arrays, since transistors in SRAM arrays stay in the steady state for most of the time. There are two major current sources for static power consumption, which are the subthreshold leakage current (off-state source to drain leakage) and the gate leakage current (gate insulator leakage) [80].

Subthreshold leakage comes from the property that the transistor switching is not ideally steep. As a result, there is still a current flow from source to drain even when the gate voltage goes below the threshold voltage ( $V_{\text {gate }}<V_{t h}$, subthreshold region). This subthreshold current can be expressed as Equation (2), where $m$ is the coupling factor between depletion layer capacitance $\left(C_{d}\right)$ and gate-oxide capacitance $\left(C_{o x}\right), k$ is the Boltzmann constant, $T$ is temperature, and $q$ is the 
electron charge:

$$
I_{d s} \propto e^{\frac{-q\left(V_{t h}-V_{g s}\right)}{m k T}}, m=1+\frac{C_{d}}{C_{o x}} \geq 1 .
$$

Subthreshold swing (SS) is the factor that shows how much gate voltage should be changed to change the $I_{d s}$ by one order. Equation (3) describes how the SS is extracted from Equation (2):

$$
S S=\frac{d V_{g s}}{d\left(\log _{10} \Delta I_{d s}\right)}=\left[\frac{d\left(\log _{10} I_{d s}\right)}{d V_{g s}}\right]^{-1}=\left[\frac{1}{\ln 10} \frac{d\left(\frac{-q\left(V_{t h}-V_{g s}\right)}{m k T}\right)}{d V_{g s}}\right]^{-1}=\frac{m k T}{q} \ln 10=m \times 60 \mathrm{mV} / \mathrm{dec} .
$$

Equations (4) and (5) show how the $V_{t h}$ and SS affect to the off state current and on state current. $\left(\frac{q}{m k T}=\frac{\ln 10}{S S}\right.$ in Equation (3)):

$$
\begin{gathered}
I_{o n}=I_{d s}\left(V_{g s}=V_{d d}\right)=\frac{1}{2} \mu_{n} C_{o x} \frac{W}{L}\left(V_{d d}-V_{t h}\right)^{2} \\
I_{o f f}=I_{d s}\left(V_{g s}=0\right) \propto e^{\frac{-q\left(V_{t h}-0\right)}{m k T}}=e^{-\frac{m n 10}{S S} V_{t h}}=10^{-\frac{V_{t h}}{S S}}=10^{-\frac{V_{t h}}{0.06 m}} .
\end{gathered}
$$

According to Equation (5), low off-state current can be achieved by high $V_{t h}$ and low $m$. $V_{t h}$ cannot be increased, because not only it is reverse to the voltage scaling but it also degrades the $I_{o n}$ as shown in Equation (4). The $I_{o n}$ degradation results in latency $\left(\tau=\frac{C V}{I_{o n}}\right)$ degradation. As a result, it is important to have low $m$, which is the coupling factor of the gate voltage and the channel potential. A coupling factor that is close to one means that the gate voltage strongly controls the channel potential. The coupling factor highly depends on the transistor geometry. Thin channels and multi-directional gates help to achieve high gate to channel potential coupling. FinFET and nanowire transistors take advantage of this.

Lower subthreshold slope allows designers to achieve same speed with lower total power consumption $\left(P=P_{d y n}+P_{\text {static }}=\alpha C V_{d d}^{2}+I_{o f f} V_{d d}\right)$ or same total power consumption with higher speed. Steep on/off behavior enables lower $I_{\text {off }}$ in the same $I_{o n}$ and $V_{d d}$ design, which results in lower static power consumption $\left(P_{\text {static }}=I_{\text {off }} V_{d d}\right)$ with constant latency $\left(\tau=\frac{C V_{d d}}{I_{o n}}\right)$ and constant dynamic power dissipation $\left(P_{d y n}=\alpha C V_{d d}^{2}\right)$. The saved static power consumption can be used to increase the dynamic power consumption and $V_{d d}$, which results in higher speed $(f=1 / \tau \propto$ $\frac{\left(V_{d d}-V_{t h}\right)^{2}}{V_{d d}}$, since $\left.\left.I_{o n} \propto\left(V_{d d}-V_{t h}\right)^{2}\right)\right)$ with constant total power consumption.

Gate leakage current becomes significant when the gate oxide thickness scales down below $2 \mathrm{~nm}$. This is because of the increase of electron quantum tunneling for the very thin gate oxide. Quantum tunneling is an effect with the particle size becomes very small like electrons, the particle can penetrate the potential barrier. This is because very small particles posses properties of a particle as well as a wave so it can penetrate the barrier as if the wave penetrates the barrier. Since electron quantum tunneling depends on the material's physical thickness, high-dielectric constant gate insulators such as hafnium oxide are already used for state of the art CMOS technology to increase the gate oxide thickness with the same gate capacitance $\left(C_{g}=\frac{\epsilon_{\mathrm{SiO}_{2}}}{t_{\mathrm{SiO}_{2}}}=\frac{\epsilon_{\mathrm{HfO}_{2}}}{t_{\mathrm{HfO}_{2}}}, \epsilon_{\mathrm{SiO}_{2}}=3.9, \epsilon_{\mathrm{HfO}_{2}}=\right.$ 25) and same electrical property.

2.2.4 Bandgap and Intrinsic Carrier Concentration. Figure 6 is the energy-band structure of a semiconductor. The energy band refers to the energy level of the electron, the conduction band is the energy band that charge carrier electrons reside, and the valence band is the energy band that charge carrier holes reside. Bandgap is the region between the conduction band and the valence band where neither the electron nor the hole exists. When the external energy, which is higher than the bandgap, is applied to an electron in the valence band, then the electron is excited to the 




Fig. 6. Energy-band structure and bandgap in semiconductor.

conduction band and the vacancy of the electron in the valence band becomes a hole. Fermi level is the highest electron energy level at $0 \mathrm{~K}$ (absolute zero temperature).

The conduction band, valence band, bandgap, and Fermi level can be compared to the sea and a wooden board. The sea level is analogous to the Fermi level, water molecules are analogous to electrons, air bubbles are analogous to holes, the wooden board is analogous to the semiconductor material, the board's thickness is analogous to bandgap, top of the board is analogous to the conduction band, bottom of the board is analogous to the valence band, and the stormy level of the sea is analogous to the temperature. Let us first explain from the Fermi level. When there is no wind and tide and the sea is calm (absolute zero temperature), there are no water molecules above the sea level and no air bubbles under the sea level. However, when there is a typhoon and the sea is stormy (room temperature), there are a bunch of water molecules above the sea level, and a bunch of water bubbles under the sea level. This is how the electrons and holes are located in comparison to the Fermi level at the absolute zero temperature and the room temperature.

Let us add the semiconductor material (floating wooden board) in the electron sea. Let us assume that the sea level is located at the middle of the wooden board. When the sea is calm (absolute zero temperature), then the board calmly floats on the sea, and there is no water above the board (no electrons in the conduction band) and there is no air bubble under the board (no holes in the valence band). However, when the sea is stormy (room temperature), then the top of the board is wet (electrons reside in the conduction band) and there are a bunch of air bubbles under the board (holes reside in the conduction band).

Impurity doping, defect states in the semiconductor, insulating behavior of the large bandgap materials can also be compared to this example. First, for the impurity doping, let us put a weight on top of the floating wooden board (n-type doping). Since the board sinks slightly more, the top of the board becomes closer to the sea level. This results in that the top of the board wets more when the sea is stormy (more electrons on the conduction band) but there are less air bubbles under the board (less holes on the valence band). If we put a helium balloon on the wooden board (p-type doping) to make it floats slightly more, then the top of the board becomes farther to the sea level. This results in that the top of the board wets less when the sea is stormy (less electrons on the conduction band) but there are more air bubbles under the board (more holes on the valence band).

Second, the defect states in the semiconductor can be compared to how firm the wooden board is. If the wooden board is very firm (high quality semiconductor materials, low defect density), then the water molecules or air bubbles cannot easily smear into the wooden board (no electrons or holes in the bandgap). But if the wooden board is soft (low quality semiconductor materials, high defect density), then water molecules or air bubbles can easily smear into the wooden board (electrons or holes in the bandgap). 
Last, the material's metallic behavior, semiconducting behavior, and insulating behavior can be compared to the thickness of the wooden board. If the wooden board is very thin, then the top of the board can easily be wet even with the mild storm (metallic behavior for narrow bandgap material). However, if the wooden board is very thick, then the top of the board cannot easily be wet even with the severe storm (insulating behavior for wide bandgap material). This also explains why the wide bandgap semiconductors have a strong endurance at high temperature and radiation (less likely be flooded during the heavy storm).

Intrinsic carrier concentration, which is the carrier concentration of a semiconductor without the impurity doping (water on top of the board and air bubbles under the board without a weight or a helium balloon), has an exponential relation to the energy bandgap and temperature as shown in Equation (6). High temperature and low bandgap (severe storm and thin wooden board) increase the intrinsic carrier concentration exponentially, which makes the semiconductor behave close to metallic (flooded board). Low temperature and wide bandgap (weak storm and thick wooden board) decrease the intrinsic carrier concentration, which makes the semiconductor behave as an insulator (dry board):

$$
n_{i} \propto e^{-\frac{E_{g}}{2 k T}},
$$

where $E_{g}$ is bandgap, $k$ is the Boltzmann constant, $T$ is temperature, and $n_{i} \sim 1.5 \times 10^{10} \mathrm{~cm}^{-3}$ for Si $27^{\circ} \mathrm{C}$.

Wide bandgap semiconductors have a strong endurance at high temperature. The reason is that low bandgap semiconductors have very high intrinsic carrier concentration and turn to metallic at high temperature. As a result, low bandgap semiconductors cannot be used as a current switch in a high-temperature environment, because there is no way to turn off the switch.

Wide bandgap semiconductors also have a strong endurance to radiation. A wider bandgap requires stronger energy to generate the electron and hole pair, so it is less likely to be disturbed by external radiation.

The low intrinsic carrier concentration of wide bandgap semiconductors makes the transistor devices have both low on-state current and off-state current. Low off-state current results in small static power dissipation but low on-state current results in long latency $\left(\tau=\frac{C V}{I_{o n}}\right)$.

2.2.5 Source and Drain Contact. Digital integrated circuits consist of billions of transistors, which are connected with metal. Because the metal-to-semiconductor contact can disturb the transistor current flow, it is important that the metal-to-semiconductor contact has low contact resistance.

The metal-to-semiconductor contact resistance in the source and the drain region can be classified into two categories: Schottky contact (left panel of the Figure 7) and Ohmic contact (right panel of the Figure 7). Schottky contact refers to the contacts where the metal work function is larger than the semiconductor work function $\left(\phi_{m}>\phi_{s}\right)$, while the Ohmic contact has the opposite relationship $\left(\phi_{m}<\phi_{s}\right)$. The work function is the energy between the Fermi level and the vacuum level, which can be seen as the energy that is required for the electron in the material to escape from the material surface to the vacuum. The metal work function is a property of the material, as is the semiconductor work function, but the latter can be changed by impurity doping.

As shown in the "in contact under equilibrium" part of Figure 7, the Ohmic contact does not disturb the current flow, since there is no energy barrier at the metal and the semiconductor interface. However, a Schottky contact is problematic to current flow because of the Schottky barrier $\left(\phi_{S B}\right)$ at the metal and the semiconductor interface. The Schottky contact resistance has an exponential relationship with the barrier height and the semiconductor doping concentration, as shown in 

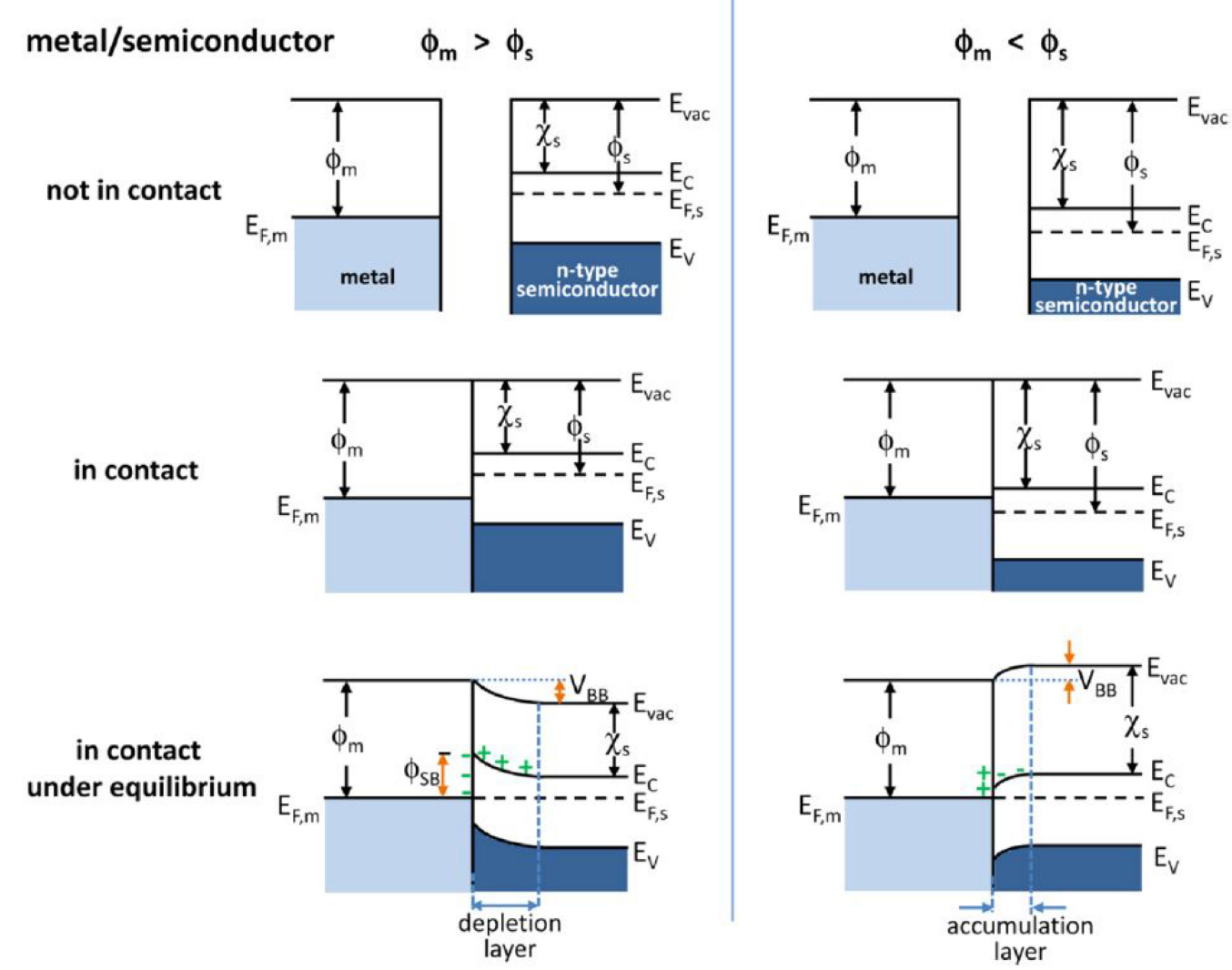

Fig. 7. Energy-band structure of the Schottky contact (left) and the Ohmic contact(right) [164]. $E_{v a c}$ is the vacuum energy level, $\phi_{m}$ is the metal work function, $\phi_{s}$ is the semiconductor work function, $\chi_{s}$ is the electron affinity of the semiconductor, and $\phi_{S B}$ is the Schottky barrier height. Reprinted (adapted) with permission from Zhang, Zhen, and Yates Jr. "Band bending in semiconductors: Chemical and physical consequences at surfaces and interfaces." Chemical reviews 112,10 (2012): 5520-5551. Copyright (2012) American Chemical Society.

Equation (7):

$$
\rho \propto \exp \left[\frac{\phi_{S B}}{\sqrt{N_{D}}}\right] .
$$

Where $\phi_{S B}$ is the barrier height in Figure 7 and $N_{D}$ is the semiconductor doping concentration. To have a low Schottky contact resistance $\rho$, it is desirable to have a low Schottky barrier $\left(\phi_{S B}=\right.$ $\left.\phi_{m}-\chi_{s}\right)$ or high doping concentration $\left(N_{D}\right)$.

\subsection{Integration Level}

2.3.1 The End of Dennard Scaling and Moore's Law. There has been a significant advancement in microprocessor technology in recent decades. Comparing the Intel 4004 processor (1971) and the recent Intel core i9-9900K processor (2018), transistor size has shrunk from $10 \mu \mathrm{m}$ to $14 \mathrm{~nm}$. Transistor count has increased from 2,250 to more than 1 billion. Throughput and frequency have increased from 0.092 MIPS and 0.74 MHz to 50,000 MIPS and 3.6 GHz. Power consumption increased from $1 \mathrm{~W}$ to $95 \mathrm{~W}$, and die size increased from $12 \mathrm{~mm}^{2}$ to $177 \mathrm{~mm}^{2}$. [36, 72, 104, 152, 




Fig. 8. Growth trend of the microprocessor [58]. Hennessy and Patterson. 2019. A new golden age for computer architecture. ACM 2019. ACM 62, 2 (Feb. 2019), 48-60. Figure 6. DOI:https://doi.org/10.1145/3282307.

Table 2. Scaling Down Rules [41]

\begin{tabular}{ccc}
\hline Scaling factor $s>1$ & Equation & Scaling \\
\hline$T_{o x}, L_{\text {gate }}, W_{\text {gate }}$ & & $1 / s$ \\
Doping concentration & & $s$ \\
Voltage & $E T_{o x}, E L_{g a t e}$ & $1 / s$ \\
Capacitance per transistor & $\frac{\epsilon_{o x} W L}{T_{o x}}$ & $1 / s$ \\
Current per transistor & $\frac{1}{2} \mu C_{o x} \frac{W}{L}\left(V_{g s}-V_{t h}\right)^{2}$ & $1 / s$ \\
Current density & $e n \mu E$ & $s$ \\
Delay & $\frac{C V}{I}$ & $1 / s$ \\
Frequency & $1 / \tau$ & $s$ \\
Transistor density & & $s^{2}$ \\
Power & $\frac{1}{2} C V^{2} f$ & $1 / s^{2}$ \\
Power density & $P A$ & 1 \\
\hline
\end{tabular}

153]. However, the performance improvement is stagnating as shown in Figure 8. The clock frequency and voltage scaling started to saturate in 2003 due to power constraints [57]. Since then, the multicore era has helped Moore's law continue, but performance improvement with multicore is also started to saturate due to its limitation from Amdahl's law [49]. Furthermore, the transistor's physical size is reaching its atomic dimension limitations.

2.3.2 Scaling Down and Its Limitations. The main goal of Moore's law and Dennard scaling is that the transistor's integration density increases and each transistor's Energy Delay Delay Product (EDDP) decreases so that processors will have more and more transistors with shorter clock period and constant power density. The cost of this improvement is the fabrication facility for finer structures. Table 2 shows how the physical properties of a transistor scale under constant electric field scaling. ( $V=E d$, where $V$ is the electric potential, $E$ is the electric field, $d$ is the distance, $E$ remains constant, $V$ and $d$ are scaled down) [41]. The constant electric field scaling has an advantage on the prevention of high electric field breakdown of the material. 
Table 3. Integration Technologies

\begin{tabular}{|c|c|c|}
\hline \multirow{3}{*}{$\begin{array}{l}\text { Dennard } \\
\text { scaling }\end{array}$} & Benefit & $\begin{array}{l}\text { Constant power density, } \times s^{2} \text { transistor density, } \times s \text { speed } \\
\text { increase }\end{array}$ \\
\hline & Limitation & $\begin{array}{l}\text { Degradation of frequency increase, growth of leakage } \\
\text { current, short channel effects }\end{array}$ \\
\hline & Improvement & $\begin{array}{l}\text { Multi directional gate structure and thin channel (FinFET } \\
\text { and nanowire) }\end{array}$ \\
\hline \multirow{4}{*}{ 3D stacking } & Benefit & $\begin{array}{l}\text { Transistor density increase, constant speed } \\
\text { Shorter interconnect: Reduce latency and energy } \\
\text { consumption } \\
\text { Denser interconnect: Increase bandwidth }\end{array}$ \\
\hline & Limitation & Power density increase \\
\hline & TSV & $\begin{array}{l}\text { Wafer-to-wafer bonding } \\
\text { Pros: No temperature constraint on upper layer } \\
\text { Cons: Wafer and via alignment issue, low via density and } \\
\text { coarse 3D integration }\end{array}$ \\
\hline & $\begin{array}{l}\text { Monolithic 3D } \\
\text { integration }\end{array}$ & $\begin{array}{l}\text { Directly fabricate upper layer on top of the lower layer } \\
\text { Pros: Denser interconnect and finer 3D integration } \\
\text { Cons: Upper layer process has temperature limitation }\end{array}$ \\
\hline \multirow[t]{2}{*}{ Lithography } & $\begin{array}{l}\text { Conventional } \\
\text { lithography }\end{array}$ & $\begin{array}{l}193 \mathrm{~nm} \text { wavelength ArF laser } \\
\text { Immersion lithography: }>40 \mathrm{~nm} \text {, use water's high } \\
\text { reflective index } \\
\text { Multiple patterning: }<40 \mathrm{~nm} \text {, litho-etch-litho-etch, } \\
\text { self-aligned double patterning }\end{array}$ \\
\hline & EUV & $13.5 \mathrm{~nm}$ wavelength comes from plasma \\
\hline
\end{tabular}

In the case of the ideal constant electric field scaling, $s^{2}$ times increase of transistor density, $s$ times increase in frequency with constant power density and die area are achieved $(s>1)$. However, this no longer holds as the device size gets smaller. The first problem is the degradation of frequency gain. Since the current is not exactly proportional to $V^{2}$ but is proportional to $\left(V-V_{t h}\right)^{2}$, frequency and voltage have this relation: $f \propto \frac{\left(V-V_{t h}\right)^{2}}{V}$. When the voltage scales down and approaches the threshold voltage, frequency gain becomes smaller than $s$. Decreasing the threshold voltage $\left(V_{t h}\right)$ together can be considered, but this will exponentially increase the subthreshold leakage current, which is proportional to $10^{-\frac{V_{t h}}{S S}}$. This results in the stagnation of the voltage scaling, and increase the power density as the transistor size gets smaller. The other problem of scaling down is short channel effects. Short channel effects occur when the channel length gets shorter, and these effects deteriorate the gate controllability of $I_{d s}$ (drain to source current). There are several kinds of short channel effects, like punch through, drain induced barrier lowering, velocity saturation, channel length modulation, high field effect, and gate-induced drain leakage as shown in Figure 9. It is very important to suppress the short channel effects under a certain level.

Short channel effects are about the parasitic current conduction and modulation of $I_{d s}$ into $V_{d s}$ when the transistor size shrinks. Punch through is the parasitic current conduction between the source and the drain inside the channel body, which is controlled by $V_{d s}$, not $V_{g s}$ (see Figure 9(a)). Drain-induced barrier lowering (DIBL) is the decrease in $V_{t h}$ when the channel length is very short, because $V_{d s}$ lowers the channel barrier height (see Figure $9(\mathrm{~b})$ ). Velocity saturation is $I_{o n}$ 


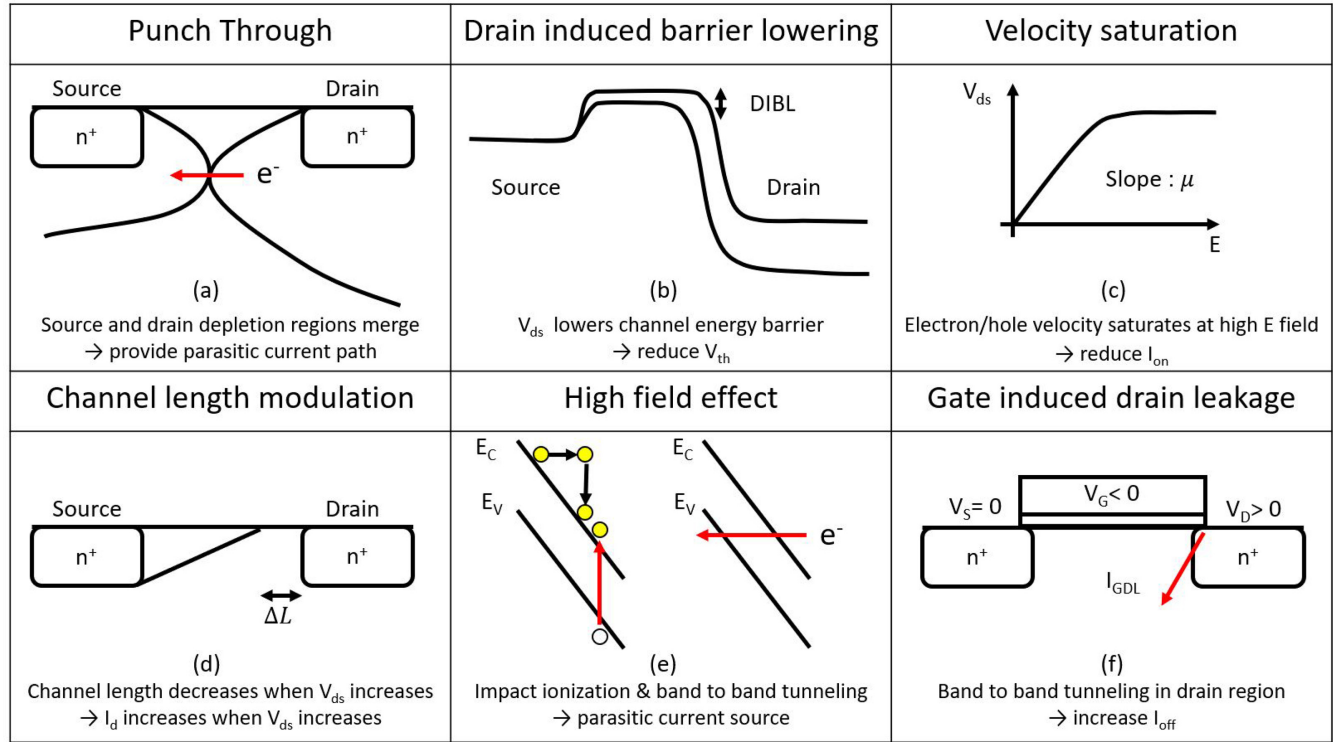

Fig. 9. Short channel effects [147].

reduction because of the mobility decrease, which is caused by charge carrier velocity saturation under the high electric field (see Figure 9(c)). Channel length modulation is that $I_{d s}$ increases when $V_{d s}$ increases due to channel length reduction (see Figure 9(d)). High field effect is the parasitic current caused by impact ionization and band-to-band tunneling (see Figure 9(e)). Gate-induced drain leakage (GIDL) is the leakage current caused by the high electric potential (voltage) difference between the gate and the source when the transistor is turned off [147] (see Figure 9(f)).

To summarize, constant electric field scaling provides free benefits in transistor integration density and speed without power density trade-off, but the voltage scaling limitation due to the offstate current and short channel effects are the barriers to further scaling.

2.3.3 Gate Structure: Mitigating Short Channel Effects and Maintaining Gate Controllability. The geometrical structure of $\mathrm{Si}$ CMOS has been evolved to suppress the short channel effects and sustain strong gate controllability. The geometrical structure has been changed from planar FET $\rightarrow$ Silicon on insulator $\rightarrow$ FinFET and is predicted to move to the gate-all-around nanowire structure [110] (see Figures 10(a)-10(d)). The more the gate surrounds the channel (multi-directional gate) and the thinner the channel depth, short channel effects are suppressed and gate controllability is enhanced [34]. A multi-gate structure increases the coupling between the gate voltage and the channel potential. A thin channel removes the parasitic current conduction path as shown in Figure 10(e).

2.3.4 Further Scaling of Nanowire Structure. A nanowire (or nanosheet) transistor, which has a gate all around (GAA) structure, is the ultimate geometry of a transistor for the suppression of short channel effects and strong gate controllability. The horizontal (or lateral) nanowire transistor and the vertical nanowire transistor are suggested for future technology (see Figure 11).

The horizontal nanowire transistor (see Figure 11(b)) has a similar structure to the FinFET (see Figure 11(a)), but FinFET's fins are replaced with the stacks of the horizontal nanowires. This has stronger gate controllability and better short channel effects suppression than FinFET while maintaining the same on-state current by stacking multiple nanowires. The vertical nanowire transistor 


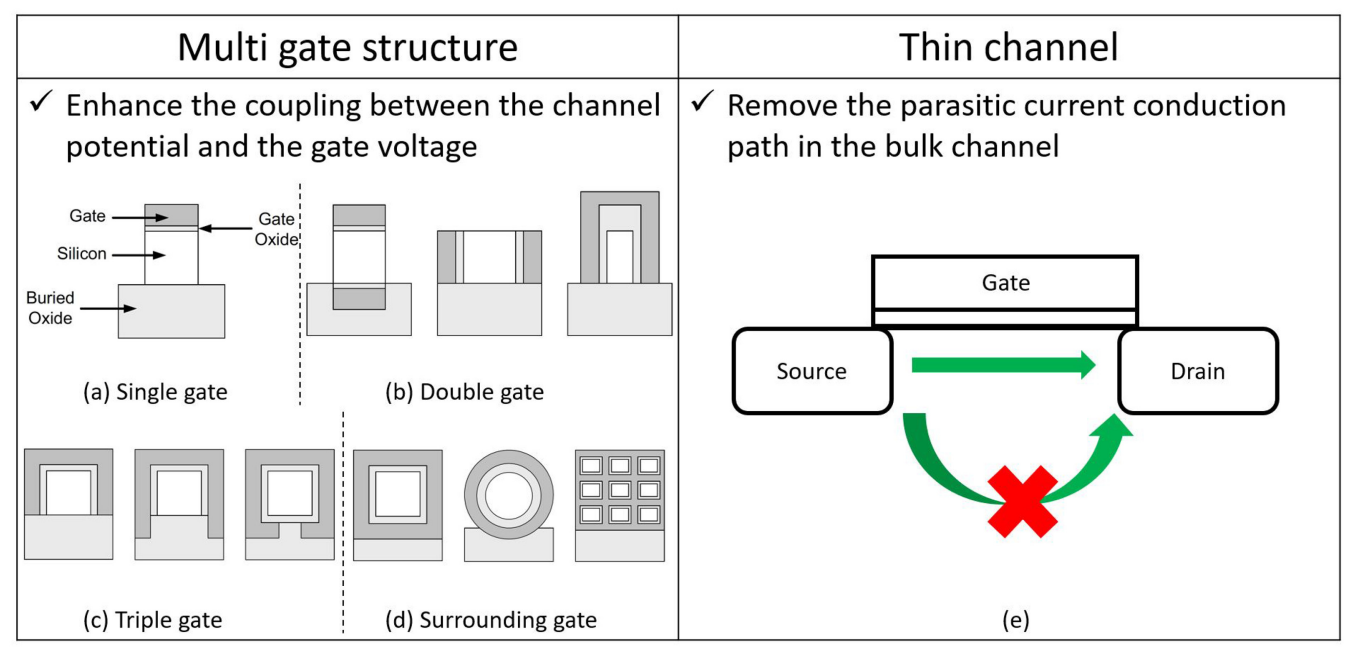

Fig. 10. Advantages of multi-directional gate structures [34] and thin channel to suppress short channel effects. (a-d) Reprinted from Microelectronic Engineering, Vol 84, Issue 9-10, Colinge, "Multi-gate SOI MOSFETS,” pp. 2071-2076, Copyright (2007), with permission from Elsevier.

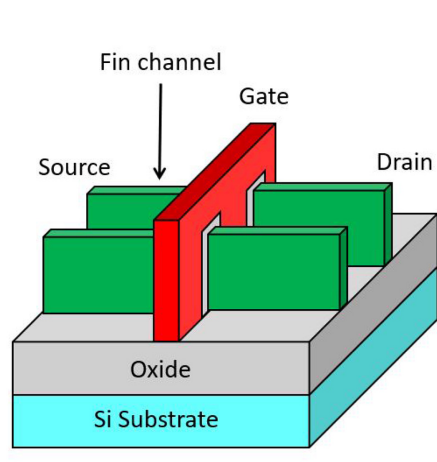

(a)



(b)

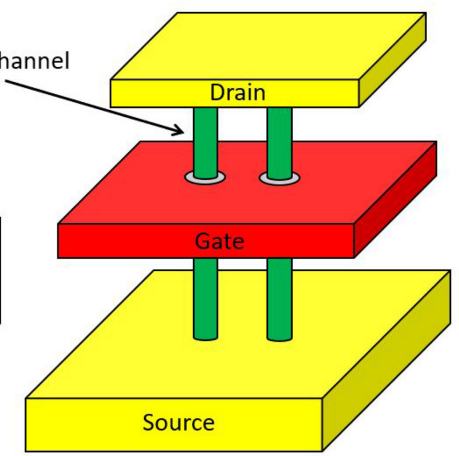

(c)

Fig. 11. (a) FinFET. (b) Horizontal nanowire transistor. (c) Vertical nanowire transistor.

(see Figure 11(c)) is suggested for the later generation to further reduce the metal to metal pitch and increase the transistor integration density. Since the source and the drain are located in different metal layers, a vertical nanowire transistor is more suitable for SRAM than complicated logic design.

2.3.5 3D Stacking. Three-dimensional vertical stacking is a way to increase the transistor density and provide denser and shorter interconnect between chips than two-dimensional integration. The shorter interconnect wire reduces interconnect latency and energy consumption, and the denser interconnect wire increases bandwidth [15, 64, 101, 124]. However, tight thermal management is required, since power density increases as the transistor density increases in 3D integration. The denser interconnect layer also contributes to increasing the power density and elevated temperatures leading to faster aging and higher fault rates. Aging-aware fault-tolerant 


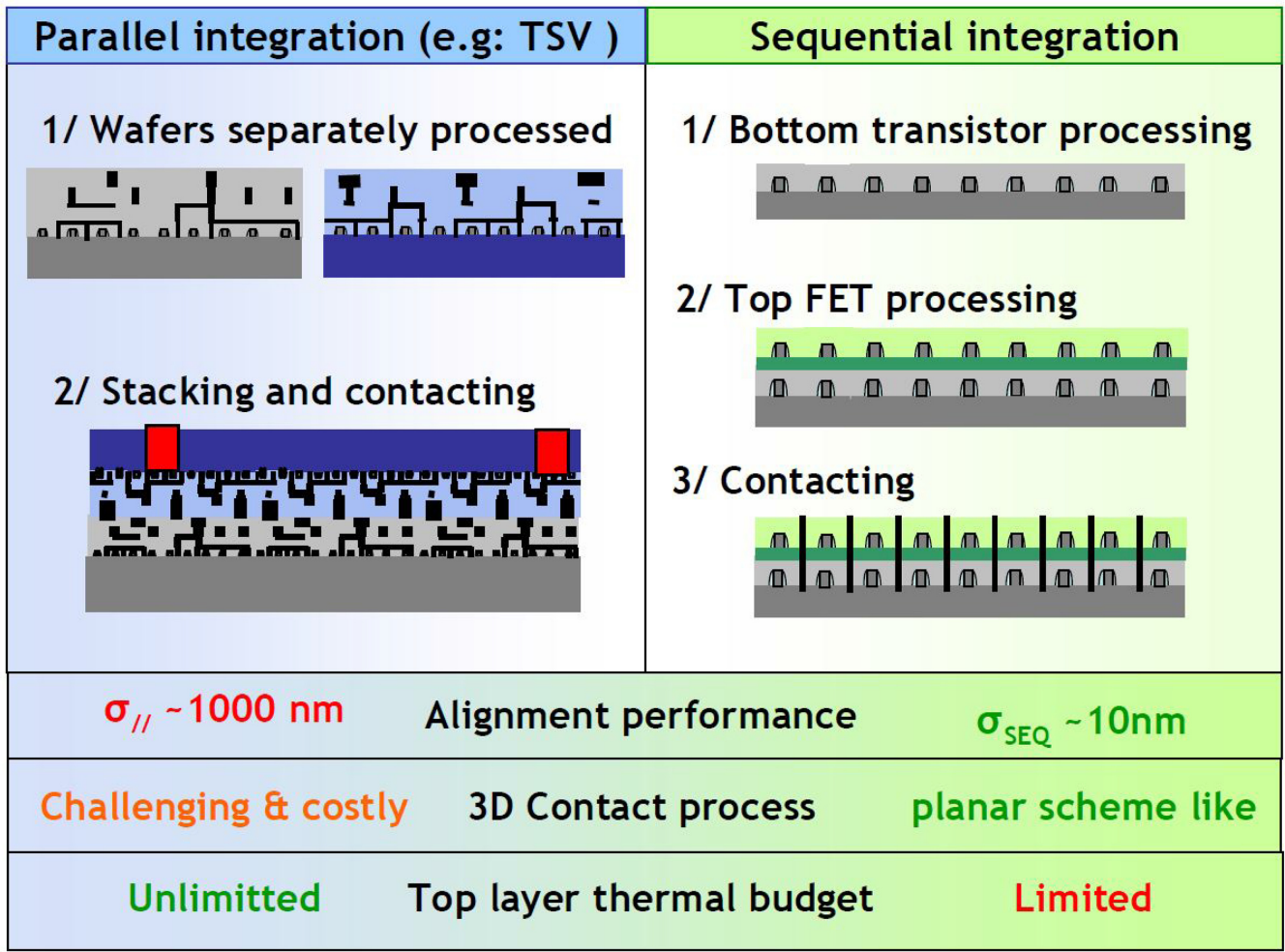

Fig. 12. 3D integration: TSV and monolithic 3D integration [13]. 2011 IEEE. Reprinted, with permission, from Batude et al., "Advances, challenges and opportunities in 3D CMOS sequential integration." 2011 International Electron Devices Meeting. IEEE, 2011.

architectures has been tailored to manage the reliability and yield problems for future 3D processors $[11,12]$.

3D stacking can be divided into through silicon via (TSV) and monolithic 3D integration [13], and their properties are summarized in Figure 12. TSV is the wafer-to-wafer bonding. Wafers are processed separately, and they are stacked and connected after each wafer is fabricated. The advantage of TSV is that the upper-layer process does not have the temperature constraint so that conventional Si process can be used. The disadvantage of TSV is that due to the wafer and via alignment issue, the via to via distance is limited to $\mu \mathrm{m}$ level, which results in low via density and coarse 3D integration [13, 149].

However, monolithic 3D integration directly fabricates the upper layer on top of the lower layer. This provides denser interconnect and finer 3D integration than TSV. In the extreme case, n-type FET and p-type FET of an inverter logic can be located on different layers [3, 13, 149]. However, the disadvantage of the monolithic 3D integration is that the upper layer fabrication process has a temperature constraint. Since the upper-layer process should not affect the lower layer's back end, the process temperature is limited to $400^{\circ} \mathrm{C}$ [71] which is far lower than the Si CMOS process temperature, which is $1,100^{\circ} \mathrm{C}$ [135]. Low temperature process technologies such as a carbon nanotube transistor, resistive random access memory, or Ge nanowire have a potential for monolithic 3D integration. 


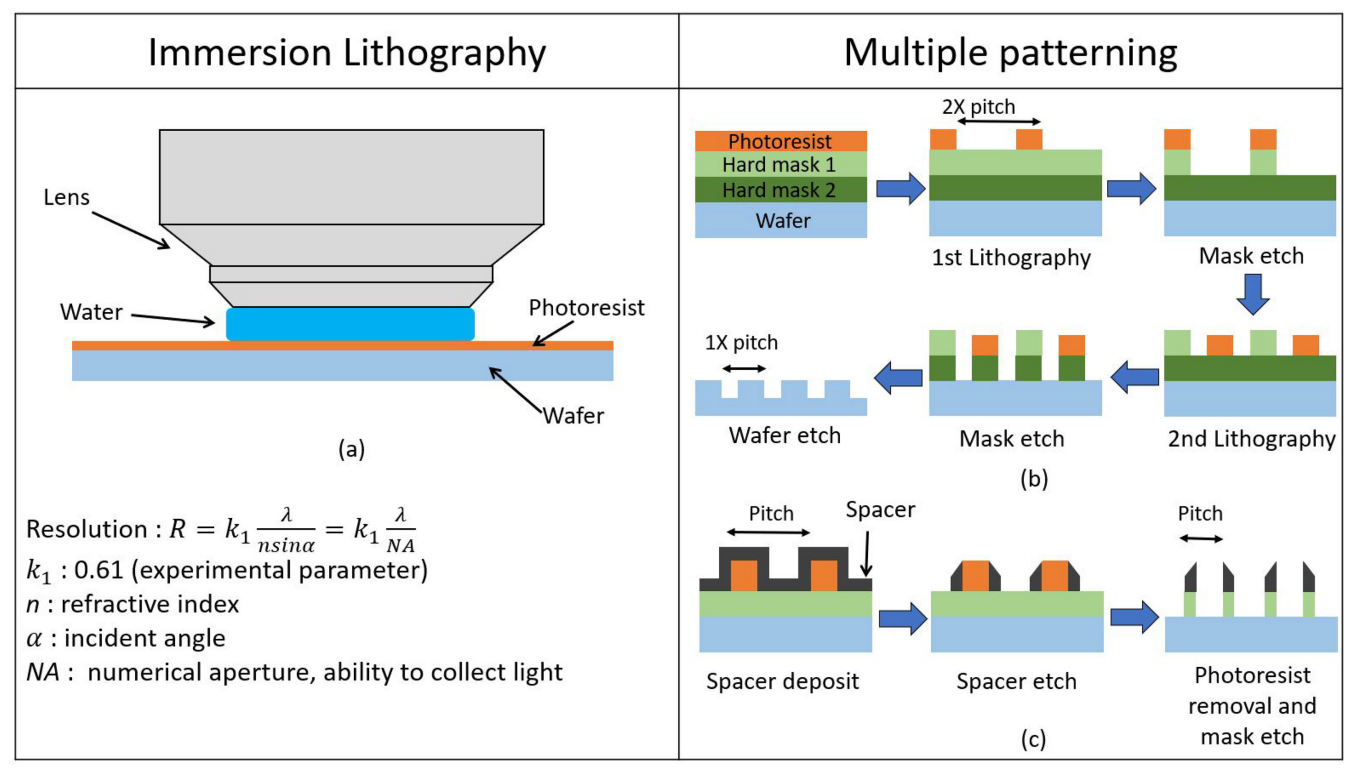

Fig. 13. Lithography techniques for finer patterning resolution.

2.3.6 EUV and Multiple Patterning. Lithography patterning is one of the main issues for scaling. TSMC and Samsung announced that they are manufacturing $7 \mathrm{~nm}$ chips by using extreme ultraviolet (EUV) technology. EUV technology uses $13.5 \mathrm{~nm}$ wavelength light, which is a lot shorter than the conventional lithography's $193 \mathrm{~nm}$ wavelength light [148]. Conventional lithography was used until the $10 \mathrm{~nm}$ process. The pattern size is really small compared to the lithography wavelength, so there have been many techniques for resolution improvement as shown in Figure 13.

Immersion lithography (see Figure 13(a)) utilizes water's higher reflective index than air's reflective index to improve the pattern resolution [148]. Below $40 \mathrm{~nm}$ technology, multiple patterning technologies such as litho-etch-litho-etch (see Figure 13(b)) or self-aligned-double-patterning (see Figure 13(c)) have been used in addition to the immersion lithography [48].

The drawback of the multiple patterning is that the number of masks increases as the pattern size decreases. Forty masks are required for 40/45 nm technology, but 80 85 masks are expected to be used in $7 \mathrm{~nm}$ technology [71, 87]. This causes issues such as the long process time, high manufacturing cost, and variation accumulation between lithography steps. Extreme ultraviolet (EUV) technology has been adopted to reduce these issues.

EUV lithography uses $13.5 \mathrm{~nm}$ wavelength light, which comes from xenon (Xe) or tin (Sn) plasma excited by a $\mathrm{CO}_{2}$ laser. This is different from the conventional lithography that uses 193 $\mathrm{nm}$ ArF laser. $\mathrm{A} \mathrm{CO}_{2}$ laser excites $25 \mu$ m diameter Xe or Sn droplets to the plasma, and the plasma emits the EUV light. The collector, which consists of multilayer mirrors, collects the emitted light. The collected light passes the scanner, which has a reflective patterning mask and finally hits the wafer. EUV uses the reflective mask, which is different from the conventional lithography's refractive optics, since there are no optical materials that are transparent to EUV wavelength light [130]. By using EUV, M1 metal layer of $7 \mathrm{~nm}$ technology can be patterned with a single mask. From $5 \mathrm{~nm}$, it is expected that multiple patterning will again be required [140]. The structure of EUV lithography is shown in Figure 14. 




Fig. 14. EUV lithography.

\section{CONDITIONS FOR EMERGING DEVICES}

From the above transistor analysis, we can figure out which conditions and aspects that one emerging device should satisfy to be used as an alternative to Si CMOS.

- high mobility

- low leakage current

- high on/off current ratio

- low subthreshold swing

- high gate controllability and negligible short channel effects

- high switching speed

- low process tempetature for monolithic 3D integration

- low manufacturing cost and high manufacturing yield

High mobility is required for high on-state current, which is important to achieve low latency without increasing dynamic power consumption. Low leakage current is important for static power saving, especially for SRAM arrays, since most of the SRAM cell transistors are in steady state for most of the time. High on/off current ratio means that the transistor has short latency due to the high on-state current and low static power consumption due to the low off-state current. Low subthreshold swing is also important for power saving and further $V_{d d}$ scaling. High gate controllability means that the short channel effects are properly controlled so that the gate voltage strongly controls the source to drain current flow. High switching speed means that the transistor should change its state from on to off or off to on rapidly. Even though one certain device has high mobility, high on-state current, steep subthreshold slope but takes $1 \mathrm{~ms}$ to turn on, that device cannot be used in high-speed processors.

Manufacturing aspects should also be considered. It will be cost effective if the fabrication process of the emerging device is compatible with the Si CMOS process. Low fabrication temperature $\left(<400^{\circ} \mathrm{C}\right)$ enables the monolithic 3D integration on top of the Si CMOS or other emerging devices. 
Table 4. Categories of Emerging Devices

\begin{tabular}{|c|c|c|}
\hline \multirow{4}{*}{ CMOS extension } & New extension & $\begin{array}{l}\text { Near-threshold computing } \\
\text { Cryogenic computing }\end{array}$ \\
\hline & New structure & $\begin{array}{c}\text { FinFET } \\
\text { Gate all around FET } \\
\text { Horizontal nanowire } \\
\text { Vertical nanowire }\end{array}$ \\
\hline & New materials & $\begin{array}{c}\text { III-V } \\
\text { Carbon nanotube } \\
\text { Ge nanowire } \\
\text { Transition metal dichalcogenide } \\
\text { Graphene nanoribbon }\end{array}$ \\
\hline & 3D stacking & $\begin{array}{c}\text { TSV } \\
\text { Monolithic integration }\end{array}$ \\
\hline \multirow{5}{*}{ Beyond CMOS } & $\begin{array}{l}\text { New transport } \\
\text { mechanism }\end{array}$ & $\begin{array}{c}\text { TFET } \\
\text { Single-electron transistor } \\
\text { Molecular electronics } \\
\text { Quantum cellular automata }\end{array}$ \\
\hline & New gating mechanisms & $\begin{array}{c}\text { NCFET } \\
\text { Ferroelectric switch }\end{array}$ \\
\hline & New state variables & Spintronics \\
\hline & New memory & $\begin{array}{c}\text { RRAM } \\
\text { PCM } \\
\text { STT-MRAM } \\
\text { FeRAM } \\
\text { FeFET } \\
\text { Memristor devices }\end{array}$ \\
\hline & New computing & DNA-based electronics \\
\hline Specific applications & High temperature & $\mathrm{SiC}$ \\
\hline
\end{tabular}

The emerging devices should have a decent material quality (defect density) so that it can have the device size and yield comparable to the Si CMOS technology.

\section{CATEGORIES OF EMERGING DEVICES}

Emerging devices can be categorized based on their features and properties as shown in Table 4 . The categories are (1) CMOS extension (thermionic emission modulation), (2) Beyond Si CMOS devices (Beyond-Boltzmann transistors), and (3) Specific applications. Si CMOS extension devices have a similar current flow mechanism as current Si CMOS, but their geometrical structures or materials are different. Beyond Si CMOS devices have novel current flow mechanism than current Si CMOS devices. Specific application devices have a similar current flow mechanism and physics as Si CMOS, but their material property makes them suitable for certain applications, such as high temperature and high radiation. The next section discusses further details of each emerging device. The detailed descriptions of near-threshold computing [44], cryogenic computing [21, 89, 108], single-electron transistors (SET) [122], molecular electronics [8], quantum cellular automata 




Fig. 15. Two-dimensional electron gas of III-V material transistor and carbon nanotube field effect transistor [97, 119]. 2005 IEEE. Reprinted, with permission, from Lin et al., "High-performance carbon nanotube fieldeffect transistor with tunable polarities." IEEE transactions on nanotechnology 4.5 (2005): 481-489. (d, e, f) Republished with permission of IOP Publishing, Ltd, from "Relation between conduction property and work function of contact metal in carbon nanotube field-effect transistors," Nosho, Ohno, Kishimoto, and Mizutani, Vol 17, Issue 14 (2006); permission conveyed through Copyright Clearance Center, Inc.

(QCA) [82], ferroelectric switch, spintronics, and DNA-based electronics [92] are beyond the scope of this article.

\section{EMERGING DEVICES' PROPERTY AND THEIR APPLICATION}

This section introduces various emerging devices such as III-V material transistor, carbon-based devices, Ge nanowire transistor, tunneling field effect transistor, negative capacitance transistor, silicon-carbide (SiC) electronics, and two-dimensional transition metal dichalcogenide transistor. Their characteristics, advantages, disadvantages, and possible applications are explained based on the previously described transistor physics.

\subsection{III-V Materials}

III-V materials consist of a 1:1 ratio of group III and group V elements, unlike Si or Ge, which are group IV elements. Boron (B), aluminum (Al), gallium (Ga), and indium (In) are group III elements, and nitrogen $(\mathbf{N})$, phosphorus $(\mathbf{P})$, arsenic (As), and antimony $(\mathbf{S b})$ are group V elements that are used as III-V compound semiconductors. Figure 15(a) shows the design of the III-V material transistor.

III-V materials have advantages of high electron mobility, low noise, and fast switching speed [109]. III-V materials' high electron mobility $\left(\sim 16,000 \mathrm{~cm}^{2} / \mathrm{Vs}\right)$ [142] comes from their different high charge carrier concentration forming mechanism than the impurity doping. As shown in Figure 15(b), the heterojunction of two different III-V materials, which have different energy bandgap, makes electrons accumulate two-dimensionally on the junction interface. As a result, the III-V material transistor has low EDDP, which enables more integration in the same power budget than Si transistor, or leads them to be used in low power applications. Wide bandgap 
III-V materials such as $\mathrm{GaN}(3.4 \mathrm{eV})$ and $\mathrm{AlGaN}(3.4 \sim 6.1 \mathrm{eV})$ can be used in power electronics or high-temperature electronics.

The disadvantages of III-V materials are difficult high quality material growth, high material growth temperature $\left(>500^{\circ} \mathrm{C}\right)[18,37$, and low pFET performance from low hole mobility [23]. Bulk III-V material is a lot more expensive to produce than $\mathrm{Si}$, which is not cost effective. Making high-quality 2D epilayer on top of the Si substrate or some other substrate is more cost effective than bulk III-V material but the epilayer growth is competitive. High growth temperature makes it difficult for III-V materials to be applied in monolithic 3D integration. Low hole mobility and pFET performance makes III-V materials difficult to be applied in CMOS logic circuits.

Several studies have been conducted to produce high quality InGaAs epilayer [39, 40]. Cointegration with SiGe pFET can compensate the low III-V pFET performance [38]. Monolithic 3D integration of III-V nFET on top of SiGe pFET is done by transferring InGaAs layer on top of the SiGe layer and conducting the low temperature transistor fabrication process after the wafer bonding [43]. III-V material's high electron mobility makes III-V material a strong candidate for high density low power data processing processors with the aid of co-integration with SiGe pFET and monolithic 3D integration. Wide bandgap III-V materials can be applied in extreme environment sensors and IoT devices.

\subsection{Carbon-based Devices}

Carbon-based devices leverage the carbon hexagonal lattice structure. Graphene and graphene nanoribbon (GNR) are two-dimensional structures, and Schottky barrier-type carbon nanotube field effect transistors (SB-type CNFETs) and MOS-type CNFETs are one-dimensional nanowire structures. In this article, carbon nanotube transistors refer to the SB-type CNFETs. MOS-type CNFETs will be discussed separately.

5.2.1 Graphene. Graphene, the two-dimensional hexagonal lattice structure of carbon, was first investigated because of its very high mobility $\left(120,000 \mathrm{~cm}^{2} / \mathrm{Vs}\right.$ theoretically, $200 \mathrm{~cm}^{2} / \mathrm{Vs}$ for after transistor fabrication of small-width graphene nanoribbons) [126] and high cut-off frequency $(400 \mathrm{GHz})$ [55]. Despite these advantages, graphene has high off-state current, since it has zero bandgap. Because of this, graphene cannot be used as a channel material for field effect transistors. Instead, carbon nanotube and graphene nanoribbons, which have high mobility and non-zero bandgap, are used as a channel material for field effect transistors.

5.2.2 Schottky Barrier-type Carbon Nanotube Field Effect Transistors. A carbon nanotube is a cylindrical rolled-up structure of a carbon hexagonal layer. A carbon nanotube transistor (see Figure 15(c)) is a nanowire transistor that has a small tube diameter $(1 \sim 2 \mathrm{~nm})$, high p-type and n-type mobility (theoretical calculation: $\sim 100,000 \mathrm{~cm}^{2} / \mathrm{Vs}[46,74]$, measured device: several hundreds to thousand $[19,74])$, high thermal conductivity, and low transfer temperature $\left(<200^{\circ} \mathrm{C}\right)$ [135]. At the sub-10-nm scale, high mobility is achieved by the ballistic transport (no scattering) in the one-dimensional channel, which means that the charge carriers swift through the carbon nanotube without any collisions or disturbance $[9,70]$. Carbon nanotube transistors use work function engineering to make n-type or p-type transistors, which is that the energy-band structure and transistor's type changes by what contact metal is used $[9,119]$. (see Figures 15(d), 15(e), and 15(f), SWNT means single-walled nanotube). Since they do not use the impurity doping to make n-type and p-type transistors, they do not degrade and fully utilize their high charge carrier mobility.

The current flow of Schottky barrier carbon nanotube transistor (SB CNFETs, in this article CNFETs refer to SB CNFETs) are controlled by the Schottky barrier thickness and quantum tunneling $[9,97]$. A Schottky barrier is the electron potential (energy) barrier at the 


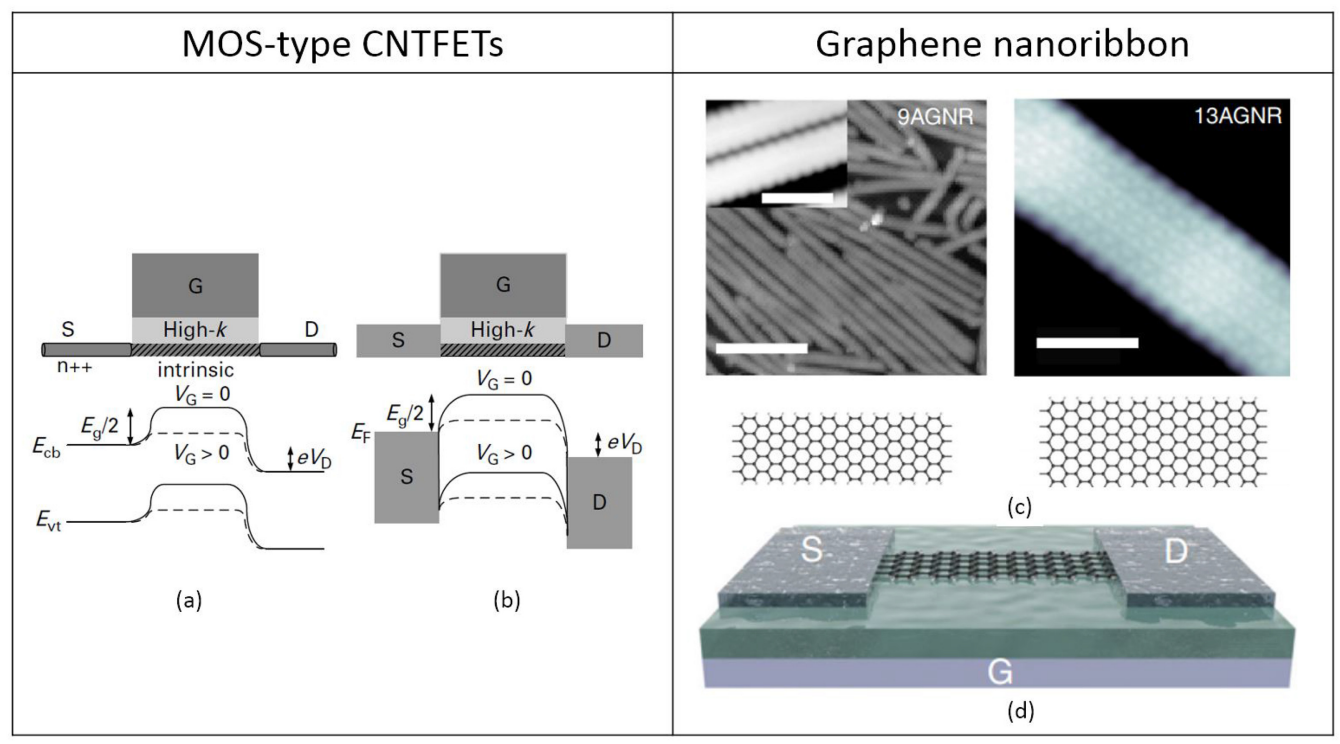

Fig. 16. (a, b) MOS-type CNFETs [156]. (c) GNR. (d) SB-type GNRFETs [100]. (c) Scale bar for 9 atoms GNR (AGNR) are $1 \mathrm{~nm}$ (up) and $10 \mathrm{~nm}$ (down), and scale bar for 13 AGNR is $2 \mathrm{~nm}$. (a, b) "Carbon nanotube and graphene device physics," by Wong and Akinwande. This version is free to view and download for private research and study only. Not for re-distribution or re-use. (c, d) By Llinas et al., "Short-channel field-effect transistors with 9-atom and 13-atom wide graphene nanoribbons." Nature communications 8,1 (2017), 1-6. Licensed under Creative Commons Attribution 4.0 International License (http://creativecommons.org/licenses/by/4.0/).

metal-semiconductor contact. The gate voltage changes the carbon nanotube's energy-band level, which changes the Schottky barrier thickness. The thickness modulation of the Schottky barrier changes the number of charge carriers that can penetrate through the Schottky barrier by quantum tunneling.

The possible application of CNFETs is in low power electronics because of its high mobility and gate-all-around structure. High mobility makes CNFETs have low latency with the same dynamic power dissipation $\left(\tau=\frac{C V}{I_{o n}}, P=C V^{2}\right)$. The gate-all-around structure enhances the gate controllability to channel potential, which results in steep on-off switching and low leakage power dissipation. CNFETs have been shown to be excellent candidates for low voltage and near threshold operations making them perfect candidates to be used in the design of sensors, IoT devices and energy-constrained devices.

Moreover, the low process temperature makes it possible for CNFETs to be used in monolithic 3D integration $[3,135]$. The high thermal conductivity helps CNFETs mitigate the power burden of $3 \mathrm{D}$ integration.

However, due to the infancy of the manufacturing process, there have been yield issues and density variations. Several studies have been conducted to mitigate these issues using new manufacturing techniques along with circuit and architectural remedies [16, 74, 121].

5.2.3 MOS-type Carbon Nanotube Field Effect Transistors. MOS-type CNFETs are another type of CNFETs, whose source and drain contacts are Ohmic contacts as shown in Figures 16(a) and 16(b). Unlike the SB-type CNFETS, the current flow mechanism of MOS-type CNFETs is the 
thermionic emission modulation, wherein the channel energy barrier controls the source to drain current flow such as the usual Si MOSFETs.

The advantage of MOS-type CNFETs over SB-type CNFETs is that they have better offstate current blocking and higher on-state current conductance, because there is no Schottky barrier in the source and the drain contact $[9,156]$. This means MOS-Type CNFETs have lower leakage current, higher on-state current, and lower Subthreshold Swing than SB-type CNFETs.

The disadvantage of MOS-type CNFETs over SB-type CNFETs is that the Ohmic contact is harder to fabricate than the Schottky contact. In case of CNFETs with doped CNT Ohmic contacts (see Figure 16(a)), the doping control in nanoscale devices is very difficult. This causes dopant number and position fluctuation that results in transistor performance variation [9]. In case of CNFETs with Ohmic metallic contacts (see Figure 16(b)), because the metal work function is fixed, it is difficult to fabricate (as detailed in Section 2.2.5). Also, n-type MOS-type CNFETs have p-type SB behavior, which can increase the leakage current [127]. As a result, SB-type CNFETs are preferred for large scale digital integrated circuits [156].

5.2.4 Graphene Nanoribbons. Graphene has high electron mobility, but because it has zero bandgap, it cannot be used as field effect transistors [28]. GNR are suggested to overcome the zero bandgap of the graphene. Graphene nanoribbons are graphenes whose widths are very narrow, with widths ranging from tens of nanometers to several nanometers as shown in Figure 16(c). Graphene nanoribbons have non-zero bandgap and their energy bandgap has an inverse relationship with the GNR width [138]. As the graphene width decreases, the two-dimensional structure is confined to a one-dimensional structure, which has different conduction band and valence band structure than the two-dimensional graphene and results in non-zero bandgap [28].

Schottky barrier-GNRFETs (SB-GNRFET) are similar to SB-CNFETs, except that the channel material is the GNR as shown in Figure 16(d). Since SB-GNRFETs do not require impurity doping, they can utilize the high electron mobility of graphene and do not suffer dopant variation [53]. As a result, SB-GNRFETs have lower EDP than Si-CMOS. SB-GNRFETs have EDP that is $2.5 \%$ of Si CMOS's EDP in the ideal case and $68 \%$ for the non-ideal case [53]. Non-ideal cases include process variations such as line-edge roughness, which are the disadvantages of GNRFETs [53].

\subsection{Ge Nanowire Transistor}

A Ge nanowire transistor is a gate-all-around transistor that has a diameter around $10 \sim 20 \mathrm{~nm}$. It has high p-type mobility, and low process temperature $\left(<420^{\circ} \mathrm{C}\right)[25,102]$. Ge nanowire achieves high hole concentration by Fermi energy pinning rather than impurity doping, so both high mobility and high hole concentration can be achieved together (see Figure 17(a)). Fermi energy pinning is where the Ge energy band is bent because of the surface states and Si shell bond so the holes are one-dimensionally accumulated in the Ge nanowire channel $[103,120]$. In the electron sea and wooden board metaphor, let us turn a wooden bowl upside down and put it into the sea. The boundary of the wooden bowl is analogous to the Si shell, and the concave part of the bowl is analogous to the Ge nanowire. Since the bowl is upside down, there is an airpocket (hole gas) in the bowl (Ge nanowire channel). A Ge nanowire with very thin $(\sim 2 \mathrm{~nm})$ Si shell has high hole concentration $\left(10^{16} \sim 10^{19} \mathrm{~cm}^{-3}\right)$ with high mobility $\left(\sim 750 \mathrm{~cm}^{2} / \mathrm{Vs}\right)[25,162]$, which outperforms SiGe strained PMOS [159].

Ge nanowire and CNT are similar in the aspect of the nanowire structure, high carrier mobility without impurity doping, and low process temperature, but they also have several different characteristics. Ge nanowire has a bigger diameter than CNT, and it can be grown directly on the Si 


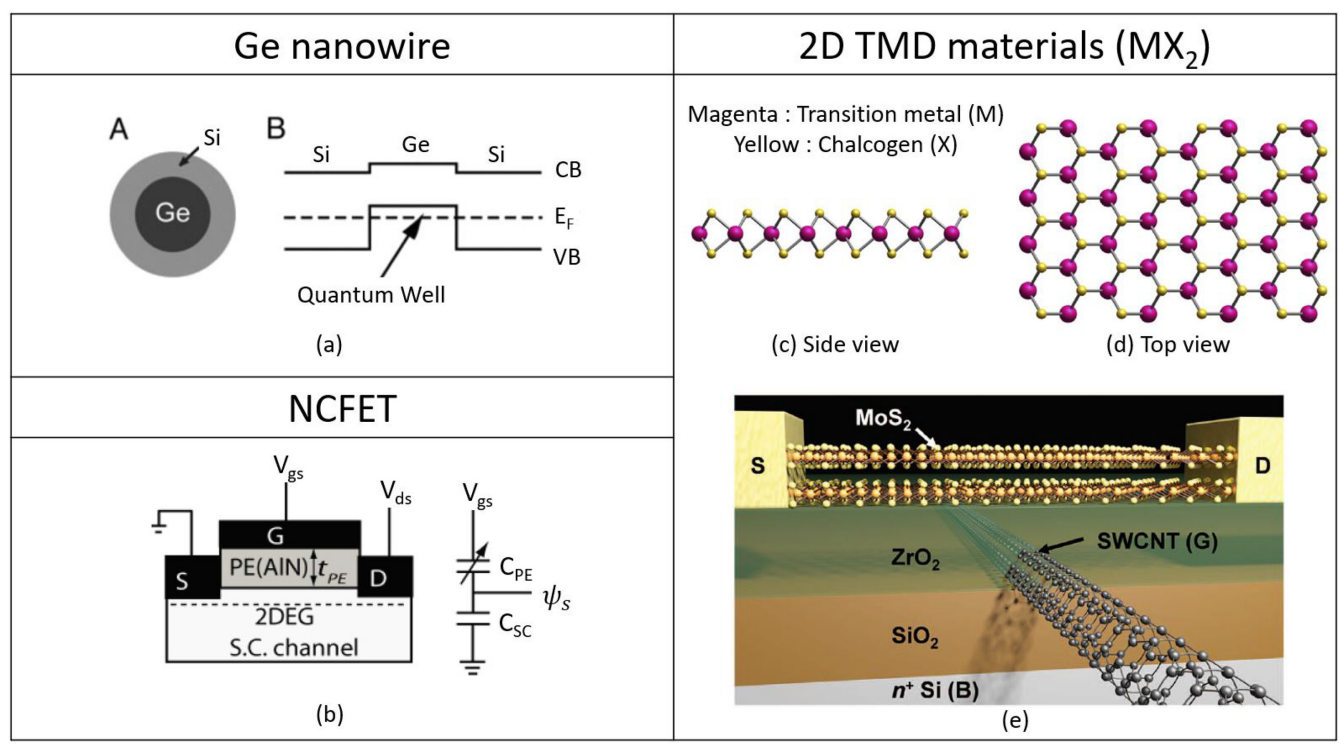

Fig. 17. One-dimensional hole gas of Ge nanowire transistor [102], NCFET [77], and two-dimensional TMD transistor [42, 47]. (a) Republished with permission of IOP Publishing, Ltd, from "Semiconductor nanowires," Lu and Lieber, Vol. 39, Issue 21, (2006); permission conveyed through Copyright Clearance Center, Inc. (b) 2013 IEEE. Reprinted, with permission, from Jana, Snider, and Jena. "Sub-Boltzmann transistors with piezoelectric gate barriers.” 2013 Third Berkeley Symposium on Energy Efficient Electronic Systems (E3S). IEEE, 2013. (c, d) Reprinted from Monatomic Two-Dimensional Layers, D'angelo and Matsuda, "Basics and Families of Monatomic Layers: Single-layer 2D Materials,” pp. 3-22 (2019), with permission from Elsevier. (e) From Desai et al. "MoS2 transistors with 1-nanometer gate lengths." Science 354.6308 (2016), 99-102. Reprinted with permission from AAAS.

substrate with a low-temperature process $[120,161]$, which has an advantage on yield, alignment, and manufacturing cost.

The low process temperature and high hole mobility of the Ge nanowire transistor makes the Ge nanowire transistor a strong candidate for monolithically 3D integrated complementary FET when co-integrated with high electron mobility transistors such as Si nanowire transistors, III$\mathrm{V}$ material transistors, or n-type Ge nanowire transistors. The narrow bandgap of Ge nanowire results in high off-state current for Ge nanowire transistor, so monolithically 3D integrated Ge nanowire circuits are more suitable for high density arithmetic computing components rather than SRAM.

\subsection{TFET}

A Tunneling Field Effect Transistor (TFET) uses a band-to-band tunneling mechanism for current flow to achieve below $60 \mathrm{mV} / \mathrm{dec}$ subthreshold swing [31]. The source-channel-drain doping is $p^{+}-p-n$ to form the tunnel junction, which is slightly different from MOSFET's $n-p-n$ structure.

TFET's steep on/off current switching and below $60 \mathrm{mV} / \mathrm{dec}$ subthreshold swing provides further room for $V_{t h}$ and $V_{d d}$ scaling, since the off-state current remains the same for lower $V_{t h}$ $\left(I_{o f f} \propto 10^{-\frac{V_{t h}}{S S}}\right.$ from Equation (5), SS is the subthreshold swing) and the frequency will remain the same for lower $V_{d d}$ and lower $V_{t h}\left(f \propto \frac{\left(V-V_{t h}\right)^{2}}{V}\right)$. The availability of further $V_{d d}$ and $V_{t h}$ 
scaling makes the TFET suitable for low voltage operations. Low voltage operation makes TFET a good candidate for not only low power electronics but also heterogeneous multicore and heterodevice core architectures, which can provide more room for EDDP optimization [54, 143].

The drawback for the TFET is its low on-state current $(10 \sim 100 \mu \mathrm{A} / \mu \mathrm{m})$. Hence, there has been further research to improve the TFET's on-state current. By using direct bandgap materials or lower bandgap materials such as GaSb/InAs, on-state current higher than Si TFET has been achieved [7, 85, 91].

\section{$5.5 \quad$ NCFET}

A negative Capacitance FET has a subthreshold swing lower than $60 \mathrm{mV} / \mathrm{dec}$. They achieve this by using the negative differential gate capacitance property of the piezoelectric aluminium nitride (AIN). A Piezoelectric material occurs when externally applied mechanical stress makes extra charges, and vice versa. Negative differential gate capacitance $\left(C_{P E}\right)$ amplifies the coupling between gate voltage and channel potential, which results in making the coupling factor $m<1$ and the subthreshold slope $\mathrm{SS}<60 \mathrm{mV} / \mathrm{dec}[77,158]$. The relationship between the negative capacitance $\left(C_{P E}\right)$, coupling factor $(m)$, and the SS are shown in Equation (8) and Figure $17(\mathrm{~b})$ :

$$
\Delta \Psi_{s}=\frac{1}{1+\frac{C_{S C}}{C_{P E}}}=\frac{1}{m} \Delta V_{g s}, m=\frac{\partial V_{g s}}{\partial \psi_{s}}, S S=\left[\frac{d\left(\log _{10} I_{d s}\right)}{d V_{g s}}\right]^{-1}=\frac{\partial V_{g s}}{\partial \psi_{s}} \frac{\partial \psi_{s}}{\partial \log _{10}\left(I_{d s}\right)}=m \times 60 \mathrm{mV} / \mathrm{dec} .
$$

Negative gate capacitance is achieved by the electrostriction effect $[77,158]$. For piezoelectric materials, when an external electric field is applied, compressive stress is created inside the material and forces further reduction in material thickness. The reduction of the material thickness will enhance the external electric field intensity. Hence, there is strong positive feedback that results in an inductive like behavior $\left(C_{P E}<0\right)$ as shown in Equation (9), where $Q$ is the effective charge stored in the system, $V$ is the applied voltage, $C_{P E}$ is the effective capacitance of the overall system, and $\alpha_{f}$ is the feedback factor [158]):

$$
Q=\left(\frac{C_{0}}{1-\alpha_{f} C_{0}}\right) V=C_{P E} V
$$

The advantage of the NCFET is the same as that of the TFET. The steep on/off current switching and below $60 \mathrm{mV} /$ dec subthreshold swing makes the NCFET one of the strong candidates for low power and low voltage electronics.

The low switching speed ( $\mu$ s to ms range) of the NCFET is an obstacle for high-speed integrated circuit applications [96]. However, there has recently been reported research that enhanced the switching speed of the NCFET to the GHz level by using Si-doped hafnia [6, 84].

\subsection{High-temperature SiC}

$\mathrm{SiC}$ is one of the prospective candidates for high-temperature applications, such as space exploration, downhole oil exploration, and automobile/aircraft engine room $[1,2,60,68,115]$. The wide bandgap of $\mathrm{SiC}(3.2 \mathrm{eV}$ versus $1.1 \mathrm{eV}$ for $\mathrm{Si}$ ) enables it to maintain its semiconductor property up to $600^{\circ} \mathrm{C}$, which is much higher than Si's temperature margin of below $300^{\circ} \mathrm{C}$ [115]. SiC's wide bandgap also allows $\mathrm{SiC}$ transistors to have low leakage current in high-temperature and highvoltage environments. SiC's high thermal conductivity makes $\mathrm{SiC}$ transistors have good thermal dissipation.

$\mathrm{SiC}$ has already been widely used in power electronics, which are high power and high voltage analog circuits $[2,163]$. When used in digital circuits, SiC is a strong candidate for hightemperature and high-radiation extreme environment sensors and processors. 
However, there are challenges for adopting $\mathrm{SiC}$ in digital integrated circuits. First, the inferior material quality and high fabrication cost compared with Si make the transistor size in $\mu \mathrm{m}$ size $[81,113]$. Furthermore, the imbalance between electron and hole mobility is more significant than $\mathrm{Si}$, which results in large $W_{p} / W_{n}$ ratio (pFET and nFET channel width ratio) for $\mathrm{SiC}$ CMOS $[60,81]$. The severe imbalance of $W_{p} / W_{n}$ ratio increases the circuit layout area.

\subsection{Two-dimensional TMD Materials}

Two-dimensional atomically thin materials such as graphene, $\mathrm{MoS}_{2}$, and other chalcogenides are the candidates for display circuits, flexible displays, and electronics. Their availability of ultimate thickness scaling in the atomic level makes them have the potential to be used in extremely scaled-down transistors, which have a channel thickness of around $1 \mathrm{~nm}$ (see Figure 17(e)).

Graphene was first investigated for two-dimensional material devices, but as we mentioned earlier, graphene has high off-state current due to its zero bandgap. To address this concern, we introduce transition metal dichalcogenide (TMD) material family $\left(\mathrm{MX}_{2}, \mathrm{M}\right.$ is $\mathrm{Mo}$ or $\mathrm{W}$. $\mathrm{X}$ is $\mathrm{S}$, $\mathrm{Se}$, or Te). The structure of TMD is a single molecular layer of X-M-X as shown in Figures 17(c) and $17(\mathrm{~d})$.

This monolayer structure facilitates the atomic level thickness control and ultimate thickness scaling. Hence, it can significantly reduce short channel effects. Stanford's group implemented a $1 \mathrm{~nm}$ gate length junctionless transistor by using CNT as a gate material, and this implementation shows the possibility for extreme-scaling [42]. The TMD monolayer on top of $\mathrm{HfO}_{2}$ has the mobility of about $200 \sim 300 \mathrm{~cm}^{2} / \mathrm{Vs}$, which is the same order as $\mathrm{Si}$. The device that has been made from TMD has 2 orders lower leakage current than a Si device [42].

TMD materials are grown by the vapor-solid growth method, and the growth temperature is $\sim 900^{\circ} \mathrm{C}$. But TMD layers can be transferred by exfoliation, which can be applied for low temperature process [95].

Low process temperature, low off-state current, extremely thin channel and material flexibility makes 2D TMD materials strong candidates for various applications such as the monolithic 3D integrated computing systems, low power memory, power-constrained electronics, and flexible electronics and sensor networks [3, 29, 151].

The implementation of TMD devices has several challenges. The high growth temperature means that TMD layers cannot directly be grown for 3D integration. The contact resistance with metal is also poor. Wafer-scale growth of high-quality films and large scale processing/manufacturing methods need to be developed [151].

\subsection{Emerging Memory Devices}

Emerging Non-Volatile Memory (ENVM) technologies, such as Phase Change Memory (PCM), spin-transfer torque magnetoresistive RAM (STT-RAM), ferromagnetic RAM (FeRAM), ferroelectric field-effect transistor (FeFET), or resistive RAM (RRAM), and so on, are some of the attractive candidates for replacements of current state-of-the-art volatile memory technologies, due to several merits such as high capacity, area efficiency, retention time, readability, reasonable read/write latencies, low voltage operation, and random access [71].

PCM is probably the most mature technology among all the ENVM technologies. It is based on the chalcogenide glasses, which can exist in both crystalline and amorphous physical states [66] (see Figure 18(a)). These physical states are used to store data, because the crystalline state has low electric resistance and the amorphous state has high electric resistance. During the read, logical one/zero is decided based on the amount of the current flowing through the PCM device. The PCM 


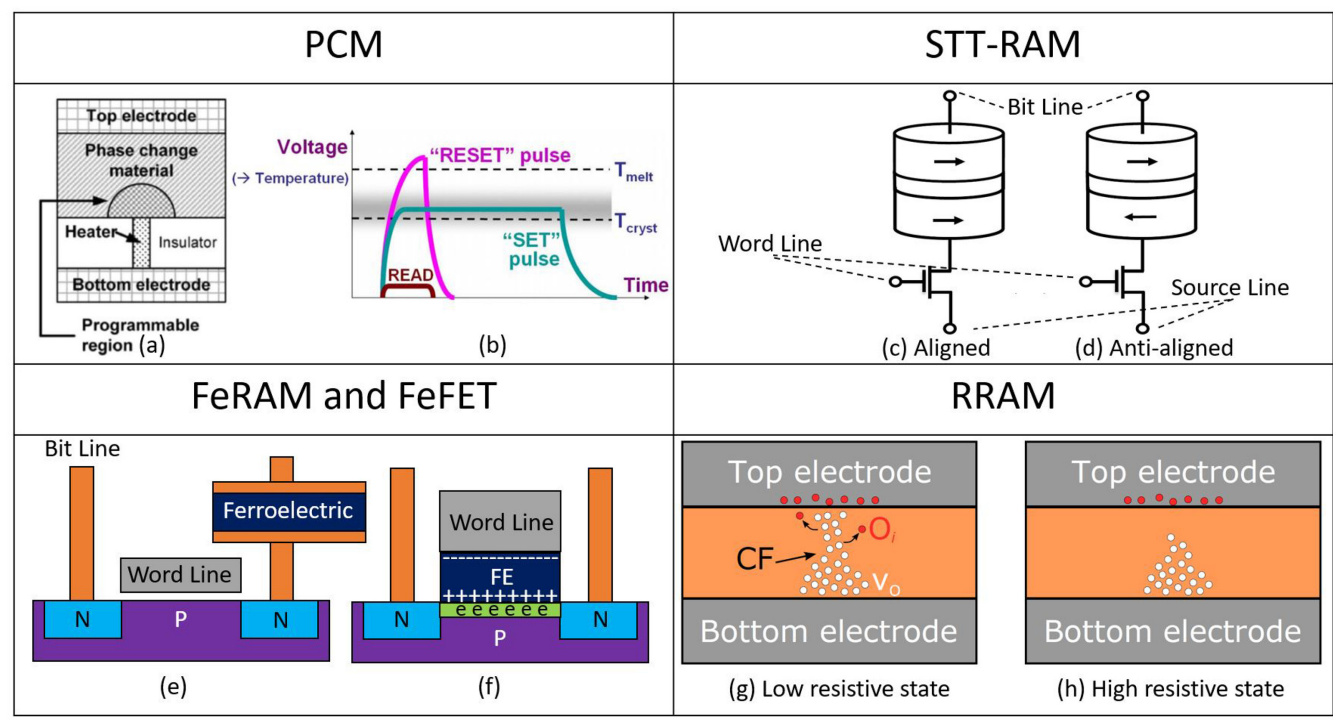

Fig. 18. Phase change memory [20, 157], STT-RAM [131], FeRAM [154], FeFET [155], and RRAM [137]. (a) 2010 IEEE. Reprinted, with permission, from Wong et al., "Phase change memory." Proceedings of the IEEE 98,12 (2010), 2201-2227. (b) Reproduced from Burr et al., "Phase change memory technology." Journal of Vacuum Science and Technology B, Nanotechnology and Microelectronics: Materials, Processing, Measurement, and Phenomena 28, 2 (2010), 223-262, with the permission of the American Vacuum Society. (c, d) 2016 IEEE. Reprinted, with permission, from Seyedzadeh et al., "Leveraging ecc to mitigate read disturbance, false reads and write faults in stt-ram." Proceedings of the 46th Annual IEEE/IFIP International Conference on Dependable Systems and Networks (DSN). IEEE, 2016. (e) Drawn by cyferz via Wikipedia creative commons and is licensed under CC BY 2.5. (https://en.wikipedia.org/wiki/Ferroelectric_RAM). (f) Drawn by cyferz via Wikipedia creative commons and is licensed under CC BY 2.5. (g, h) 2017 IEEE. Reprinted, with permission, from Sklénard et al. "Advances in the understanding of microscopic switching mechanisms in ReRAM devices." Proceedings of the 47th European Solid-State Device Research Conference (ESSDERC). IEEE, 2017 (https://en.wikipedia.org/wiki/Fe_FET).

write operation is divided into two phases, called reset and set (see Figure 18(b)). During the reset stage, a strong electric pulse is applied for a very short period of time to melt the chalcogenide glasses and cool them down quickly to achieve the amorphous state. Next, the set phase is carried out to achieve the crystalline state. During the set phase, a less stronger electric pulse is applied for a long period of time to anneal the amorphous glasses into the crystalline glasses [71].

In the past few years, researchers have explored PCM technology for building stand-alone as well as hybrid memory with DRAM [88, 125, 165]. Recently, Intel and Micron Technology announced 3D cross-point memory [22], which is faster than SSD and denser than DRAM. However, PCM also suffers from the following disadvantages. The costly write operation is an overhead in terms of long write latency (100 ns $~ 10 \mu$ s [24]) from the set stage as well as high energy (30 pJ) from the reset stage, which results in low write bandwidth [88]. For example, a $20 \mathrm{~nm} 8$ Gb PCM prototype provides some tens of $\mathrm{Mb} / \mathrm{s}$ write bandwidth [32]. High-temperature tolerance and thermal disturb are the challenges for scalability, since PCM uses thermal heating and cooling [71]. Additionally, multi-level cell (MLC) PCM suffers from resistance drifts [20, 93].

STT-RAM is an evolution in magnetic storage from hard disk drives to solid-state drives, which uses magnetic charges instead of electrical, to store data. There are two magnetic layers and one thin insulating tunnel junction layer is located between the two magnetic layers. When the two 
magnetic layers' magnetic moments are aligned (see Figure 18(c)), the tunnel junction layer has low electric resistance. However, when the two magnetic layers' magnetic moments are anti-aligned (see Figure 18(d)), the tunnel junction layer has high electric resistance. Key advantages of this technology are excellent high scalability, simpler architecture, shorter write time ( $<10 \mathrm{~ns}$ [24]), and infinite endurance (in the order of $10^{16}$ ). STT-RAM's latency is comparable to 6T-SRAM, however, STT-RAM is non-volatile and has a small footprint. However, the major challenge faced by this technology is its high programming current, which makes it difficult to integrate with standard CMOS technology [76].

RRAM is another type of the emerging non-volatile memory technology, which uses the insulator sandwiched between metal layers. Oxygen vacancies or metal cations create a filament inside the insulator, and the resistance is determined by whether the filament is formed (see Figure 18(g)) or broken (see Figure 18(h)). RRAM shares many advantages with PCM but it does not encounter the resistance drift problem and has lower write energy and higher density [20,93]. While some recent prototypes of RRAM are optimized for density trading off latency (i.e., a $32 \mathrm{~Gb}$ part from SanDisk [98] targeted as Flash replacement), this technology can also be leveraged to build low latency memories. A prototype from Micron [50] is an example of the industrial effort to hold up latency-optimized RRAM for the main memory. Also, RRAM has demonstrated reasonable endurance [90], significantly alleviating the wear-out problem in PCM. However, RRAM suffers from a data retention problem $[27,128]$, which could be mitigated using the appropriate use of the errorcorrecting code (ECC).

FeRAM design is similar to the DRAM (one transistor and one capacitor), but FeRAM uses a ferromagnetic material as a capacitor (see Figure 18(e)), instead of a dielectric material as a capacitor in DRAM design. The storage of the data in a ferromagnetic material is non-volatile. This memory is attractive, because it offers closely identical write and read latencies, in-circuit programmability, fast access time ( $100 \mathrm{~ns}[106])$, and low energy consumption. As a result, FeRAM can be used as low power, very fast flash-like memory in the embedded applications [71]. However, destructive read, high cost and low density are some of the major drawbacks of this technology [79].

FeFET is a non-volatile memory with a field-effect transistor structure. As shown in Figure 18(f), it uses ferroelectric material as a gate insulator and does not have an external capacitor. The ferroelectric material has two stable polarization states. An external electric field larger than a certain value (coercive field) can change the polarization state from one to another [107]. Applying a positive voltage pulse (electric field from gate to channel) to the gate electrode changes the polarization state to one whose polarization direction is from gate to channel as shown in Figure 18(f). As a result, channel inversion occurs and inversion electrons accumulate to the channel and the FeFET is set to a low $V_{t h}$ state [118]. Applying a negative voltage pulse changes the polarization state in the opposite direction and sets the FeFET to a high $V_{t h}$ state. The Read voltage should be smaller than the coercive voltage, making the read process non-destructive [107]. The use of hafnium zirconium oxide (HZO) $[10,26]$, doped or undoped $\mathrm{HfO}_{2}[107,118]$ allows the FeFET be CMOS compatible, since $\mathrm{HfO}_{2}$ has been already widely used for CMOS high- $\kappa$ gate dielectric. The advantages of FeFET are multifold; it has higher density than FeRAM, because it does not have an external capacitor. It also has higher speed ( $10 \mathrm{~ns}[45])$ and lower write-energy than STT-RAM, RRAM, and PCM due to the field-driven write process [10, 26, 107, 118]. Moreover, FeFET has a high $I_{\text {on }} / I_{\text {off }}$ ratio $\left(10^{4} \sim 10^{6}[26]\right)$. Last, because FeFET is CMOS process compatible, it can be used as an on-chip embedded NVM (eNVM) [10, 107]. The disadvantages of FeFET are cycling stability and statistical variations [26, 107], and its limited endurance $\left(10^{5}\right)[10,26,118]$. FeFET can be used as both an on-chip embedded NVM or non-volatile switch in digital circuits [26]. A 32 Mbit array was demonstrated in GlobalFoundries' $22 \mathrm{~nm}$ ferroelectric $\mathrm{HfO}_{2}$ fully depleted silicon on insulator (FDSOI) technology [45, 107]. A non-volatile System-on-Chip (SoC) was 


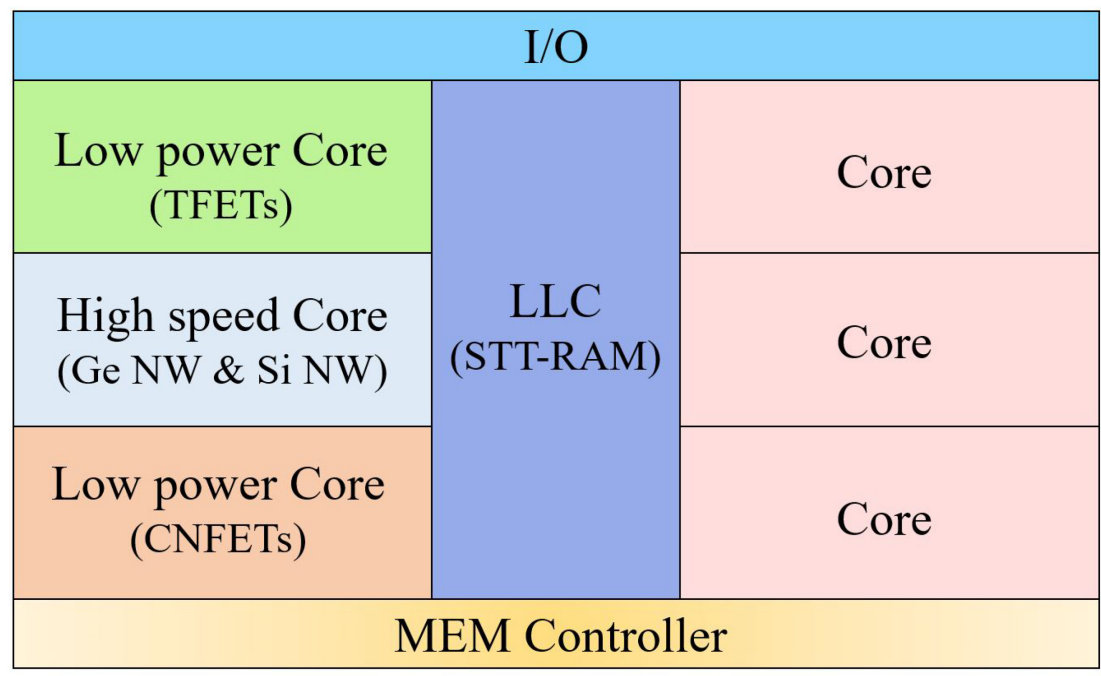

Fig. 19. Hybrid scaling.

fabricated in a $130 \mathrm{~nm}$ ferroelectric CMOS process [99]. Adopting FeFETs to FPGA components such as lookup tables (LUTs) and routing switches can save area and power [26]. FeFET's nonvolatility and CMOS compatibility also has an advantage on compute-in-memory, neuromorphic computing, and deep learning accelerator applications by minimizing data movement during the training process $[10,26,78,107,118]$.

\subsection{Emerging Materials' Physical Values and Their Perspective to the Processor Levels}

Table 5 summarizes the emerging materials properties and their device characteristics. Wide bandgap material transistors show endurance in high-temperature and high-radiation environments, and have low leakage current, but have a long latency due to their low on-state current. Their high thermal conductivity means that the device can dissipate a large amount of heat so it can have a higher power budget. Low dielectric constant $(\epsilon)$ for bulk semiconductor (not the gate insulator) means that it has low parasitic capacitance so it has small dynamic power dissipation $\left(C_{\text {parasitic }} \propto \epsilon, P_{d y n} \propto C V^{2}, C\right.$ includes $\left.C_{\text {parasitic }}\right)$. The logic type is which types of the transistor (n-type or p-type or both) can be fabricated with that material. The n-type transistor can be made when the electron conduction (conductivity $\propto$ mobility $\times$ charge carrier concentration) is good, and the p-type transistor can be made when the hole conduction is good.

High mobility results in high on-state current density, and high on-state current density results in low latency. For state of the art Si CMOS, the mobility is several hundred, the current density is $2 \mathrm{~mA} / \mu \mathrm{m}$, and the latency of the inverter and the NAND gate is several $p s$. Low subthreshold swing (steep on/off current switching) is desirable for further $V_{d d}$ scaling and low power applications. High current on/off ratio is desirable for negligible off-state power consumption and high material quality is important to suppress the defect oriented leakage current. Process temperature lower than $400^{\circ} \mathrm{C}$ makes the material suitable for monolithic 3D integration.

\section{APPLICATIONS IN PROCESSOR LEVEL}

In this section, we will take a look at how emerging devices can aid in better system-design decisions. Hybrid scaling is an approach to configure the processor with modules made of different emerging devices to take advantage of each emerging device technology (see Figure 19). For 


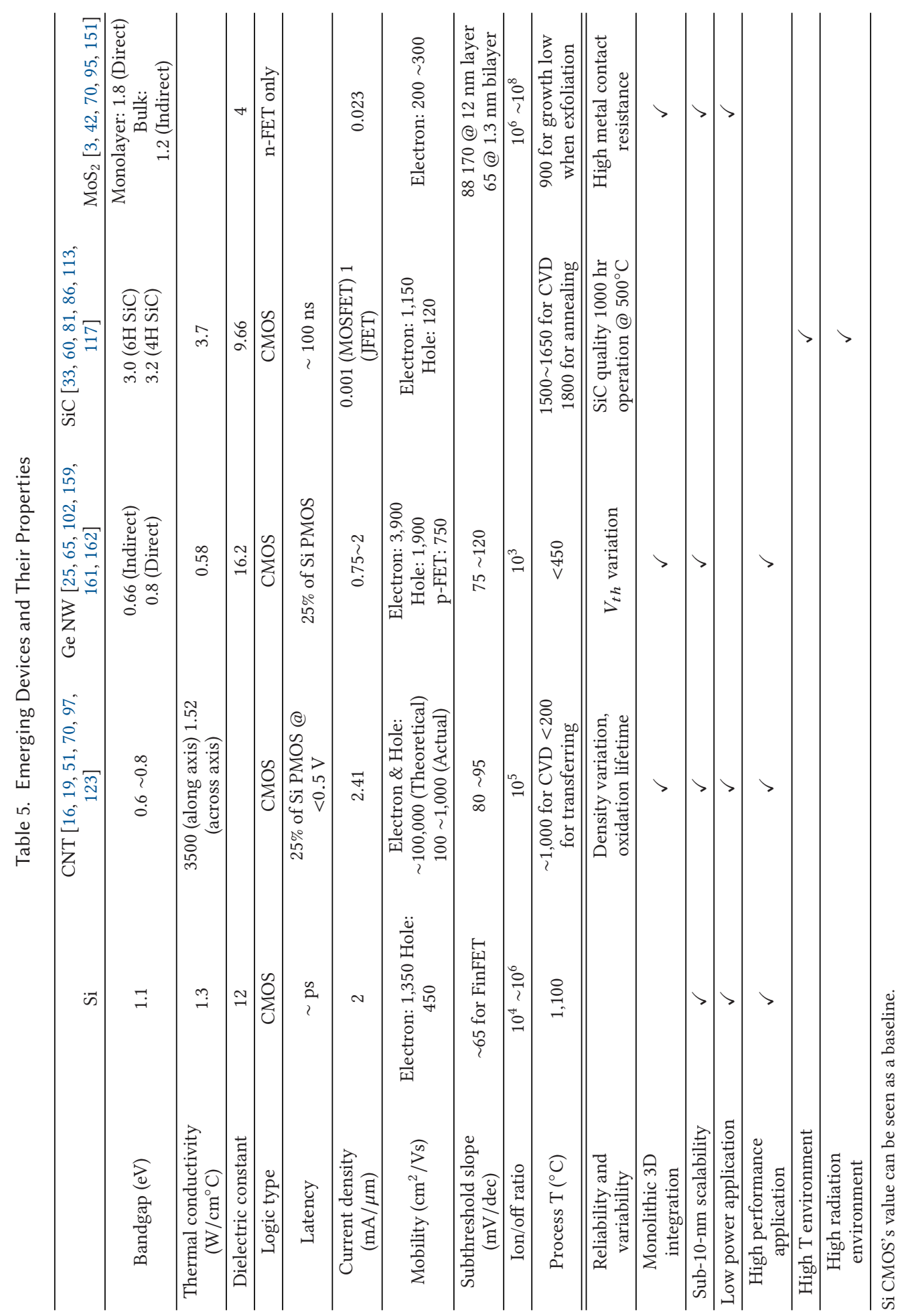


example, hybrid scaling, such as heterogeneous cores with TFET or CNFET cores, can be done for data-dominated low-power applications. The granularity of hybrid scaling can be varied. We show a few prior works that propose adopting hybrid scaling of emerging devices and 3D integration to improve performance.

\subsection{Very Large Scale Integration of CNFET-based Processor}

Carbon nanotube, which has a small diameter and gate-all-around structure, is an attractive candidate for the extremely scaled-down version of the transistor. High electron and hole mobilities result in low latency, while the high on/off current ratio results in a low off-state current. These properties make CNFET a good candidate for highly integrated, high-throughput, high-frequency, and low-power data processing and computations. It has been shown that the CNFET processor has an EDP advantage over the Si CMOS processor. In 2017, the CNFET-based RISC-V processor was reported and showed that CNFET-based processor has a $2.9 \times$ improvement in EDP at $0.4 \mathrm{~V}$ than a Si-based processor [5].

There are several challenges in realizing a large-scale integrated CNFET processor, which include material defects, manufacturing defects and variability [59]. The variability, reliability and yield issues are dealt with not only at the device and circuit level $[16,59,74,121]$ but also at the architecture level [4]. There have been several studies about implementing very large scale integration of CNFET with correct functionality. Stanford's group fabricated a 1-bit single-instruction (SUBNEG) CNFET computer with 178 CNFETs [136]. Larger scale CNFET computers were fabricated and reported in 2019, which consist of more than 14,000 complementary CNFETs, a 32-bit RISC-V instruction sets, and support 16-bit data and addresses [59]. These studies validate the realization of large scale integration of beyond silicon devices.

\subsection{D Integration and Hybrid Scaling: Further Performance Enhancement of CNFET-based Processor}

Carbon nanotube's low transfer temperature $\left(<200^{\circ} \mathrm{C}\right)$ provides the possibility of monolithic 3D integration of a CNFET system. 3D integration enables higher transistor density than 2D integration and is more energy efficient than 2D integration, because chip to chip wire length decreases so memory access latency and energy are reduced [3]. Fine grained monolithic 3D integration enables 1000 times denser vertical interconnect than TSV, which results in not only wide bandwidth between layers but also densely interwoven logic and memory components, which provides more room for EDP improvement [3]. Fine grained monolithic 3D integration with various emerging devices such as emerging memories or TMD materials provides even more room for EDP optimization. Here, we list some of the previous studies that achieve EDP enhancement with monolithic 3D integration and hybrid scaling.

Stanford group fabricated a 3D hybrid scaling of CNT sensor networks. CNFET, RRAM, and Si CMOS are monolithically integrated (see Figure 20) [135]. This sensor network takes advantage of 3D integration and emerging devices so they can sense huge amounts of data, processing them in situ, and store the highly processed data directly on-chip [135]. This research shows that the massive amount of 3D monolithic cells are fabricated with correct functionality in the wafer scale. The yield is $98.82 \%$ after testing 100,000 individual monolithic 3D cells.

Another research from the Stanford group, which is called N3XT, implemented a monolithically integrated 3D system for abundant data computing (see Figure 21) [3]. Atomically thin devices such as CNFET and 2D materials are used for computing, and emerging nonvolatile memories such as 3D RRAM and STT-MRAM are used for building the memory system. These emerging devices are 3D integrated at a fine granularity. EDP improvement of $1,000 \times$ is achieved from the contribution of architecture, transistor, and memory cells altogether. 




Fig. 20. 3D monolithically integrated CNT sensor networks [135]. Reprinted by permission from Springer Nature: Springer Nature, "Three-dimensional integration of nanotechnologies for computing and data storage on a single chip," Shulaker et al. (2017).

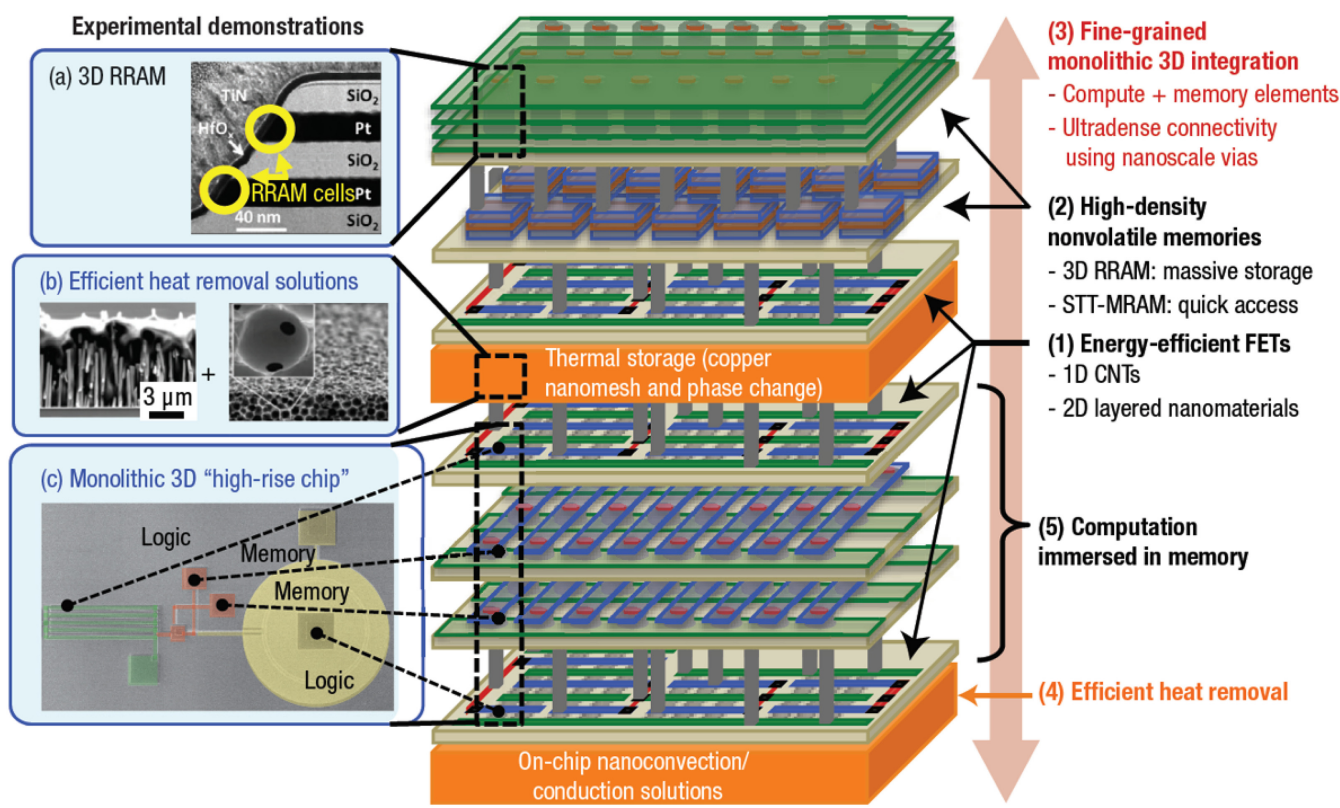

Fig. 21. Monolithically integrated 3D system for abundant data computing [3]. 2015 IEEE. Reprinted, with permission, from Aly et al., "Energy-efficient abundant-data computing: The N3XT 1,000 x." Computer 48,12 (2015), 24-33.

It was shown that CNT sensors can be integrated together with the data processing unit and memory, and this on-chip three-dimensional integration provides high bandwidth, low EDP, and in situ data processing. This research shows that integration of multiple emerging devices can improve the data collection (3D integrated CNT sensor), data transporting (on chip monolithic 3D 


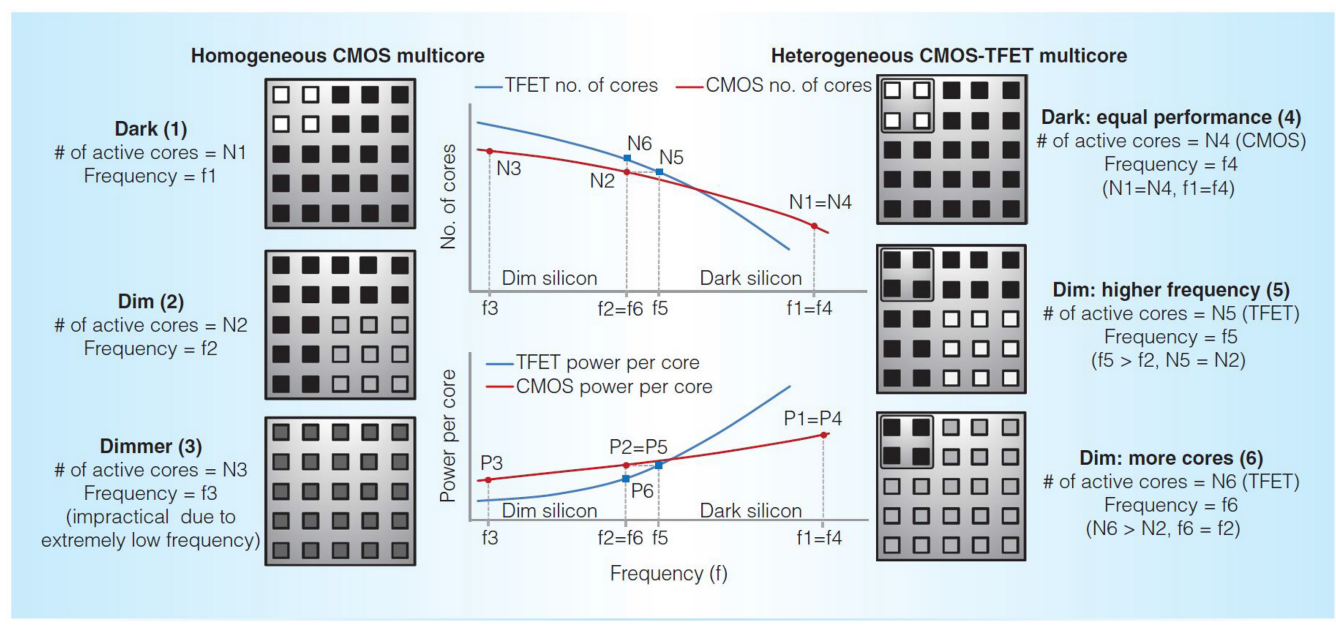

Fig. 22. Heterogeneous CMOS-TFET multicore processor [143]. 2013 IEEE. Reprinted, with permission, from Swaminathan et al., "Steep-slope devices: From dark to dim silicon." IEEE Micro 33, 5 (2013), 50-59.

integration of sensor and processing unit), data storage (3D integrated RRAM), and data processing (monolithically integrated CNFET and 2D materials) significantly.

\subsection{III-V Materials}

III-V material's high electron mobility makes it a good candidate for low power data processor with the aid of co-integration with other pFET. Digital logic components such as the inverter and the SRAM are fabricated with InGaAs nFET and SiGe pFET, which have channel length of $35 \mathrm{~nm}$ and SRAM size of $0.45 \mu \mathrm{m}^{2}$ [38]. The nFET InGaAs and pFET SiGe hybrid inverter is fabricated as monolithic 3D integration, which has InGaAs nFET top layer and SiGe pFET bottom layer [43]. The complex fabrication process of hybrid CMOS circuit makes it difficult to configure more complicated circuits. However, the previous studies show that III-V electronics can be monolithically 3D integrated at a fine granularity (single-transistor dimension), and this shows the possibility that III-V electronics can provide more room for performance optimization once they can be fabricated for complex logic components and processors.

\subsection{TFET-Si CMOS Heterogeneous Core}

TFET's steep on/off current slope and low off-state current make it a good candidate for low power applications. TFET has better EDDP than Si CMOS for the supply voltage lower than $0.5 \mathrm{~V}$ [7]. From this property, CMOS-TFET heterogeneous multicore processor has been investigated. By adopting heterogeneous thread-to-core mapping, dynamic work partitioning, dynamic power partitioning, and application-to-core mapping, this heterogeneous multicore system has $13 \sim 27 \%$ speed enhancement than Si CMOS multicore system (see Figure 22) [143]. Finer granularity provides further room for EDDP optimization. Even in the single-core processor, compositing some of the processor components with TFET and other processor components with Si-CMOS can improve the EDDP by $26 \%$ than Si CMOS only processor [54]. These studies show that emerging device heterogeneous cores for CPUs and GPUs have an advantage on low power high performance data processing because of their lower EDDP than Si only or emerging device only cores. Advancement in the fabrication process will make it possible to integrate various emerging devices with finer granularity, which will provide more room for further EDDP optimizations. 


\subsection{SiC High-temperature Electronics}

$\mathrm{SiC}$ has three times wider bandgap than $\mathrm{Si}$, which makes it endurable to high-temperature and high-radiation environment. As a result, $\mathrm{SiC}$ has been widely used for analog circuits in power electronics. However, it is important to overcome challenges such as material defects and device yield to be used as a very large scale integrated circuits in the extreme environment. There are several studies to fabricate digital IC components that correctly operate in high-temperature environment.

NASA is conducting research about configuring SiC digital circuits for high-temperature environment such as Venus lander $\left(>400^{\circ} \mathrm{C}\right)$. They have fabricated clock generator (sub $\mathrm{MHz}, 176$ JFET transistors, $\left.3 \times 3 \mathrm{~mm}^{2}\right)$ and SRAM ( $4 \times 4$ array, 195 JFET transistors) that lasts for $10,000 \mathrm{~h}$ at Venus environment, which is $460^{\circ} \mathrm{C}, 9.3 \mathrm{Mpa}$ (92 times of Earth's atmospheric pressure) with the atmospheric composition of $\mathrm{CO}_{2}, \mathrm{~N}_{2}, \mathrm{SO}_{2}, \mathrm{H}_{2} \mathrm{~S}$, and so on [112, 114, 141].

Raytheon taped out a SiC clock generator, ring oscillator, and DFF, which lasted for $100 \mathrm{~h}$ in $470^{\circ} \mathrm{C}$. Based on this, they configured SRAM and 16-bit microcontroller based on OpenMSP [60]. The microcontroller is for $470^{\circ} \mathrm{C}$ operation, the supply voltage is $12 \mathrm{~V}$ and the frequency is $0.5 \mathrm{MHz}$. A $16 \times 16 \mathrm{SiC}$ image sensor array with peripheral circuits is fabricated by using 1,959 transistors [62]. SiC process design kit is developed, and 16-bit microcontroller are fabricated with 10,000 transistors based on the PDK. The fabricated microcontroller does not work correctly, because the SRAM module does not work properly [133]. These studies show the potential that SiC electronics can conduct data collection, transporting, storage, and processing in environments where conventional Si electronics cannot be applied.

\subsection{Flexible Electronics and Monolithic 3D Integration of 2D TMD}

$\mathrm{MoS}_{2}$ 's thin and flexible nature makes it feasible for flexible electronics [29], ubiquitous electronics and display technology [151]. It has higher mobility than amorphous silicon, which has been used for flexible electronics, so $\mathrm{MoS}_{2}$ flexible electronics will provide higher frequency than amorphous silicon-based flexible electronics. Its low off-state current from atomically thin nature makes it have the potential to be applied in low power electronics. Low process temperature (when transferred from already grown substrates) enables $\mathrm{MoS}_{2}$ to be applied in monolithic 3D integration.

There are several studies that fabricated digital components and simple processors based on $\mathrm{MoS}_{2}$, that confirm they operate correctly. SRAM and a five-stage ring oscillators $(1.6 \mathrm{GHz})$ with $\mathrm{MoS}_{2}$ was implemented [151] and a 1-bit microprocessor with 115 transistors was also implemented [150]. $\mathrm{MoS}_{2}$ contributed to the EDP improvement of the processor when applied on monolithic 3D integration with the hybrid scaling [3]. The availability of flexible electronics enables data processing for a wider and more omnipresent environment, availability of monolithic 3D integration contributes to the EDP improvement, and low static power has an advantage on long battery life for IoT devices.

\subsection{Emerging Memory Technologies}

Emerging memory technologies such as resistive random access memory (RRAM), phase change memory (PCM), spin-transfer torque magnetic random access memory (STT-MRAM), ferroelectric random access memory (FeRAM) and ferroelectric field-effect transistor (FeFET) are attractive candidates for low power, high density, and non-volatile main memory design. Typically, RRAM, PCM, and STT-MRAM can be fabricated in the metal layers as part of a standard CMOS Back End of Line process. These memories generally utilize a crossbar structure, which enables an extremely dense memory array with memory cell area of $4 F^{2}$ (versus $6 F^{2}$ in DRAM) [70], where $\mathrm{F}$ is the technology feature size. The read latency of these memories is comparable to DRAM, while the 
write latency is slightly higher. Using architectural optimizations [146, 160], these memories can be used as stand-alone or side-by-side DRAM as main memory. STT-MRAM has shorter latency than RRAM and PCRAM, thus can be used not only as the main memory but also in the last level cache [83]. FeFET's non-volatility and CMOS compatibility make FeFET to be used as an eNVM.

Not only for the on-chip memory and storage, but these emerging memories can also be used as a general-purpose in-memory computing and biologically inspired computing $[10,26,69,105,144$, $145,166]$. Inspired from the bandwidth-limited interface between main memory and computation units, performing computation within the memory using in-memory computing could help alleviate this bottleneck as well as reduce the overall energy consumption of the system by reducing the data transfer $[75,78,94]$. Taking advantage of the emerging memories' memristive nature enables emerging memories to be applied in neural net and deep learning accelerators, which further improve the performance and energy efficiency $[30,132,139]$.

\section{CONCLUSION}

Beyond Si CMOS devices are often investigated to overcome the limitations of conventional $\mathrm{Si}$ CMOS devices. Emerging devices can contribute to data collection, transporting, storage, and processing. This article introduced the fundamentals and properties of emerging devices and their possible applications in system level design to non-experts. This was done to aid system designers with device level technology considerations. Basic terminologies and physics of the transistor were described first, and emerging devices such as the III-V transistor, carbon-based devices, Ge nanowire transistor, TFET, NCFET, $\mathrm{SiC}$ transistor, $\mathrm{MoS}_{2}$ transistor, and emerging memory technologies such as RRAM, PCM, STT-MRAM, FeRAM, and FeFET were then introduced and explained based on these terminologies and physics. Finally, the implications and potential applications of emerging devices and memories for future processing systems were discussed.

\section{REFERENCES}

[1] Affan Abbasi, Robert Murphree, Sajib Roy, Marvin Suggs, John Fraley, H. Alan Mantooth, Jia Di, and Tobias Erlbacher. 2019. High-temperature memory design, implementation, and characterization in $1 \mu \mathrm{m} \mathrm{SiC} \mathrm{CMOS} \mathrm{tech-}$ nology. In Proceedings of the Additional Conferences (Device Packaging, HiTEC, HiTEN, \& CICMT), Vol. 2019. International Microelectronics Assembly and Packaging Society, 000011-000015.

[2] Shamim Ahmed, Arman Ur Rashid, Md Maksudul Hossain, Tom Vrotsos, A. Matthew Francis, and H. Alan Mantooth. 2019. DC modeling and geometry scaling of SiC low-voltage MOSFETs for integrated circuit design. IEEE f. Emerg. Select. Top. Power Electron. 7, 3 (2019), 1574-1583.

[3] Mohamed M. Sabry Aly, Mingyu Gao, Gage Hills, Chi-Shuen Lee, Greg Pitner, Max M. Shulaker, Tony F. Wu, Mehdi Asheghi, Jeff Bokor, Franz Franchetti et al. 2015. Energy-efficient abundant-data computing: The N3XT 1,000 x. Computer 48, 12 (2015), 24-33.

[4] Aporva Amarnath, Javad Bagherzadeh, Jielun Tan, and Ronald G. Dreslinski. 2019. 3DTUBE: A design framework for high-variation carbon nanotube-based transistor technology. In Proceedings of the IEEE/ACM International Symposium on Low Power Electronics and Design (ISLPED'19). IEEE, 1-6.

[5] Aporva Amarnath, Siying Feng, Subhankar Pal, Tutu Ajayi, Austin Rovinski, and Ronald G. Dreslinski. 2017. A carbon nanotube transistor-based RISC-V processor using pass transistor logic. In Proceedings of the IEEE/ACM International Symposium on Low Power Electronics and Design (ISLPED'17). IEEE, 1-6.

[6] Hussam Amrouch, Girish Pahwa, Amol D. Gaidhane, Jörg Henkel, and Yogesh Singh Chauhan. 2018. Negative capacitance transistor to address the fundamental limitations in technology scaling: Processor performance. IEEE Access 6 (2018), 52754-52765.

[7] Uygar E. Avci, Daniel H. Morris, and Ian A. Young. 2015. Tunnel field-effect transistors: Prospects and challenges. IEEE 7. Electron Dev. Soc. 3, 3 (2015), 88-95.

[8] Arieh Aviram and Mark A. Ratner. 1974. Molecular rectifiers. Chem. Phys. Lett. 29, 2 (1974), 277-283.

[9] Phaedon Avouris, Zhihong Chen, and Vasili Perebeinos. 2010. Carbon-based electronics. In Nanosci. Technol.: Collect. Rev. Nature f. World Scientific, Singapore, 174-184.

[10] Ahmedullah Aziz, Evelyn T Breyer, An Chen, Xiaoming Chen, Suman Datta, Sumeet Kumar Gupta, Michael Hoffmann, Xiaobo Sharon Hu, Adrian Ionescu, Matthew Jerry et al. 2018. Computing with Ferroelectric FETs: Devices, 
Models, Systems, and Applications. In Proceedings of the Design, Automation and Test in Europe Conference and Exhibition (DATE'18). IEEE, 1289-1298.

[11] Javad Bagherzadeh, Aporva Amarnath, Jielun Tan, Subhankar Pal, and Ronald G. Dreslinski. 2020. R2D3: A reliability engine for 3D parallel systems. In Proceedings of the 57th Annual Design Automation Conference (DAC'20).

[12] J. Bagherzadeh and V. Bertacco. 2017. 3DFAR: A three-dimensional fabric for reliable multi-core processors. In Proceedings of the Design, Automation Test in Europe Conference Exhibition (DATE'17). 310-313.

[13] P. Batude, M. Vinet, B. Previtali, C. Tabone, C. Xu, J. Mazurier, O. Weber, F. Andrieu, L. Tosti, L. Brevard et al. 2011. Advances, challenges and opportunities in 3D CMOS sequential integration. In Proceedings of the International Electron Devices Meeting. IEEE, 7-3.

[14] Kerry Bernstein, Ralph K. Cavin, Wolfgang Porod, Alan Seabaugh, and Jeff Welser. 2010. Device and architecture outlook for beyond CMOS switches. Proc. IEEE 98, 12 (2010), 2169-2184.

[15] Bryan Black, Murali Annavaram, Ned Brekelbaum, John DeVale, Lei Jiang, Gabriel H. Loh, Don McCaule, Pat Morrow, Donald W. Nelson, Daniel Pantuso et al. 2006. Die stacking (3D) microarchitecture. In Proceedings of the 39th Annual IEEE/ACM International Symposium on Microarchitecture. IEEE Computer Society, 469-479.

[16] Shashikanth Bobba, Jie Zhang, Pierre-Emmanuel Gaillardon, H.-S. Philip Wong, Subhasish Mitra, and Giovanni de Micheli. 2014. System level benchmarking with yield-enhanced standard cell library for carbon nanotube VLSI circuits. ACM J. Emerg. Technol. Comput. Syst. 10, 4 (2014), 33.

[17] Mark T. Bohr and Ian A. Young. 2017. CMOS scaling trends and beyond. IEEE Micro 37, 6 (2017), 20-29.

[18] Mattias Borg, Heinz Schmid, Kirsten E Moselund, Davide Cutaia, and Heike Riel. 2015. Mechanisms of templateassisted selective epitaxy of InAs nanowires on Si. J. Appl. Phys. 117, 14 (2015), 144303.

[19] Gerald J. Brady, Austin J. Way, Nathaniel S. Safron, Harold T. Evensen, Padma Gopalan, and Michael S. Arnold. 2016. Quasi-ballistic carbon nanotube array transistors with current density exceeding Si and GaAs. Sci. Adv. 2, 9 (2016), e1601240.

[20] Geoffrey W. Burr, Matthew J. Breitwisch, Michele Franceschini, Davide Garetto, Kailash Gopalakrishnan, Bryan Jackson, Bülent Kurdi, Chung Lam, Luis A. Lastras, Alvaro Padilla et al. 2010. Phase change memory technology. F. Vac. Sci. Technol. B, Nanotechnol. Microelectron.: Mater., Process., Meas. Phenom. 28, 2 (2010), 223-262.

[21] Ilkwon Byun, Dongmoon Min, Gyu-hyeon Lee, Seongmin Na, and Jangwoo Kim. 2020. CryoCore: A fast and dense processor architecture for cryogenic computing. In Proceedings of the ACM/IEEE 47th Annual International Symposium on Computer Architecture (ISCA'20). IEEE, 335-348.

[22] Hernan A. Castro. 2016. Accessing memory cells in parallel in a cross-point array. U.S. Patent 9,312,005.

[23] Robert S. Chau. 2008. Emerging device nanotechnology for future high-speed and energy-efficient VLSI: Challenges and opportunities. In Proceedings of the 38th European Solid-State Device Research Conference (ESSDERC'08). IEEE, $1-3$.

[24] An Chen. 2016. A review of emerging non-volatile memory (NVM) technologies and applications. Solid-State Electron. 125 (2016), 25-38.

[25] Lin Chen, Fuxi Cai, Ugo Otuonye, and Wei D. Lu. 2015. Vertical Ge/Si core/shell nanowire junctionless transistor. Nano Lett. 16, 1 (2015), 420-426.

[26] Xiaoming Chen, Xiaoyu Sun, Panni Wang, Suman Datta, Xiaobo Sharon Hu, Xunzhao Yin, Matthew Jerry, Shimeng Yu, Ann Franchesca Laguna, Kai Ni et al. 2019. The impact of ferroelectric FETs on digital and analog circuits and architectures. IEEE Design Test 37, 1 (2019), 79-99.

[27] Yang Yin Chen, Robin Degraeve, Bogdan Govoreanu, Sergiu Clima, Ludovic Goux, Andrea Fantini, Gouri Sankar Kar, Dirk J Wouters, Guido Groeseneken, and Malgorzata Jurczak. 2013. Postcycling LRS retention analysis in HfO 2/Hf RRAM 1T1R device. IEEE Electron. Device Lett. 34, 5 (2013), 626-628.

[28] Zhihong Chen, Yu-Ming Lin, Michael J. Rooks, and Phaedon Avouris. 2007. Graphene nano-ribbon electronics. Phys. E: Low-dimension. Syst. Nanostruct. 40, 2 (2007), 228-232.

[29] Rui Cheng, Shan Jiang, Yu Chen, Yuan Liu, Nathan Weiss, Hung-Chieh Cheng, Hao Wu, Yu Huang, and Xiangfeng Duan. 2014. Few-layer molybdenum disulfide transistors and circuits for high-speed flexible electronics. Nature Commun. 5 (2014), 5143.

[30] Ping Chi, Shuangchen Li, Cong Xu, Tao Zhang, Jishen Zhao, Yongpan Liu, Yu Wang, and Yuan Xie. 2016. Prime: A novel processing-in-memory architecture for neural network computation in reram-based main memory. In $A C M$ SIGARCH Computer Architecture News, Vol. 44. IEEE Press, 27-39.

[31] Woo Young Choi, Byung-Gook Park, Jong Duk Lee, and Tsu-Jae King Liu. 2007. Tunneling field-effect transistors (TFETs) with subthreshold swing (SS) less than $60 \mathrm{mV} /$ dec. IEEE Electron. Device Lett. 28, 8 (2007), 743-745.

[32] Youngdon Choi, Ickhyun Song, Mu-Hui Park, Hoeju Chung, Sanghoan Chang, Beakhyoung Cho, Jinyoung Kim, Younghoon Oh, Duckmin Kwon, Jung Sunwoo et al. 2012. A 20nm 1.8 V 8Gb PRAM with 40MB/s program bandwidth. In Proceedings of the IEEE International Solid-State Circuits Conference. IEEE, 46-48. 
[33] David T. Clark, Ewan P. Ramsay, A. E. Murphy, Dave A. Smith, Robin Thompson, R. A. R. Young, Jennifer D. Cormack, C. Zhu, S. Finney, John Fletcher et al. 2011. High-temperature silicon carbide CMOS integrated circuits. In Materials Science Forum, Vol. 679. Trans Tech Publications, 726-729.

[34] Jean Pierre Colinge. 2007. Multi-gate soi mosfets. Microelectron. Eng. 84, 9-10 (2007), 2071-2076.

[35] Jean-Pierre Colinge. 2012. Junctionless transistors. In Proceedings of the IEEE International Meeting for Future of Electron Devices. IEEE, 1-2.

[36] Ian Cutress. 2018. The Intel 9th Gen Review: Core i9-9900K, Core i7-9700K and Core i5-9600K Tested. Retrieved November 3, 2019 from https://www.anandtech.com/show/13400/intel-9th-gen-core-i9-9900k-i7-9700k-i5-9600kreview.

[37] L. Czornomaz, N. Daix, P. Kerber, K. Lister, Daniele Caimi, C. Rossel, Marilyne Sousa, E. Uccelli, and Jean Fompeyrine. 2013. Scalability of ultra-thin-body and BOX InGaAs MOSFETs on silicon. In Proceedings of the European Solid-State Device Research Conference (ESSDERC'13). IEEE, 143-146.

[38] L. Czornomaz, V. Djara, V. Deshpande, E. O’Connor, M. Sousa, D. Caimi, K. Cheng, and J. Fompeyrine. 2016. First demonstration of InGaAs/SiGe CMOS inverters and dense SRAM arrays on Si using selective epitaxy and standard FEOL processes. In Proceedings of the IEEE Symposium on VLSI Technology. IEEE, 1-2.

[39] L. Czornomaz, E. Uccelli, M. Sousa, V. Deshpande, V. Djara, D. Caimi, M. D. Rossell, R. Erni, and J. Fompeyrine. 2015. Confined epitaxial lateral overgrowth (CELO): A novel concept for scalable integration of CMOS-compatible InGaAs-on-insulator MOSFETs on large-area Si substrates. In Proceedings of the Symposium on VLSI Technology (VLSITechnology'15). IEEE, T172-T173.

[40] N. Daix, E. Uccelli, L. Czornomaz, D. Caimi, C. Rossel, M. Sousa, H. Siegwart, C. Marchiori, J. M. Hartmann, K.-T. Shiu et al. 2014. Towards large size substrates for III-V co-integration made by direct wafer bonding on Si. APL Mater. 2, 8 (2014), 086104.

[41] Robert H. Dennard, Fritz H. Gaensslen, V. Leo Rideout, Ernest Bassous, and Andre R. LeBlanc. 1974. Design of ion-implanted MOSFET's with very small physical dimensions. IEEE f. Solid-State Circ. 9, 5 (1974), 256-268.

[42] Sujay B. Desai, Surabhi R. Madhvapathy, Angada B. Sachid, Juan Pablo Llinas, Qingxiao Wang, Geun Ho Ahn, Gregory Pitner, Moon J. Kim, Jeffrey Bokor, Chenming Hu et al. 2016. MoS2 transistors with 1-nanometer gate lengths. Science 354, 6308 (2016), 99-102.

[43] V. Deshpande, V. Djara, E. O’Connor, P. Hashemi, K. Balakrishnan, M. Sousa, D. Caimi, A. Olziersky, L. Czornomaz, and J. Fompeyrine. 2015. Advanced 3D monolithic hybrid CMOS with sub-50 nm gate inverters featuring replacement metal gate (RMG)-InGaAs nFETs on SiGe-OI fin pFETs. In Proceedings of the IEEE International Electron Devices Meeting (IEDM'15). IEEE, 8-8.

[44] Ronald G. Dreslinski, Michael Wieckowski, David Blaauw, Dennis Sylvester, and Trevor Mudge. 2010. Nearthreshold computing: Reclaiming Moore's law through energy efficient integrated circuits. Proc. IEEE 98, 2 (2010), 253-266.

[45] S. Dünkel, Martin Trentzsch, Regina Richter, Patrick Moll, Christine Fuchs, Oliver Gehring, Mateusz Majer, Stefan Wittek, B. Müller, Thomas Melde et al. 2017. A FeFET-based super-low-power ultra-fast embedded NVM technology for 22nm FDSOI and beyond. In Proceedings of the IEEE International Electron Devices Meeting (IEDM'17). IEEE, 19-7.

[46] T. Dürkop, S. A. Getty, Enrique Cobas, and M. S. Fuhrer. 2004. Extraordinary mobility in semiconducting carbon nanotubes. Nano Lett. 4, 1 (2004), 35-39.

[47] Marie D'angelo and Iwao Matsuda. 2019. Basics and families of monatomic layers: Single-layer 2D materials. In Monatomic Two-Dimensional Layers. Elsevier, 3-22.

[48] Semiconductor Engineering. [n.d.]. Multiple patterning. Retrieved from https://semiengineering.com/knowledge centers/manufacturing/patterning/multipatterning/.

[49] Hadi Esmaeilzadeh, Emily Blem, Renee St. Amant, Karthikeyan Sankaralingam, and Doug Burger. 2011. Dark silicon and the end of multicore scaling. In Proceedings of the 38th Annual International Symposium on Computer Architecture (ISCA'11). IEEE, 365-376.

[50] Richard Fackenthal, Makoto Kitagawa, Wataru Otsuka, Kirk Prall, Duane Mills, Keiichi Tsutsui, Jahanshir Javanifard, Kerry Tedrow, Tomohito Tsushima, Yoshiyuki Shibahara et al. 2014. 19.7 A 16Gb ReRAM with 200MB/s write and $1 \mathrm{~GB} / \mathrm{s}$ read in $27 \mathrm{~nm}$ technology. In Proceedings of the IEEE International Solid-State Circuits Conference Digest of Technical Papers (ISSCC'14). IEEE, 338-339.

[51] Aaron D. Franklin, Mathieu Luisier, Shu-Jen Han, George Tulevski, Chris M. Breslin, Lynne Gignac, Mark S. Lundstrom, and Wilfried Haensch. 2012. Sub-10 nm carbon nanotube transistor. Nano Lett. 12, 2 (2012), 758-762.

[52] John Gantz and David Reinsel. 2012. The digital universe in 2020: Big data, bigger digital shadows, and biggest growth in the far east. IDC iView: IDC Anal. Future 2007, 2012 (2012), 1-16.

[53] Morteza Gholipour, Ying-Yu Chen, Amit Sangai, Nasser Masoumi, and Deming Chen. 2015. Analytical SPICEcompatible model of Schottky barrier-type GNRFETs with performance analysis. IEEE Trans. Very Large Scale Integr. Syst. 24, 2 (2015), 650-663. 
[54] Bhargava Gopireddy, Dimitrios Skarlatos, Wenjuan Zhu, and Josep Torrellas. 2018. HetCore: TFET-CMOS heterodevice architecture for CPUs and GPUs. In Proceedings of the ACM/IEEE 45th Annual International Symposium on Computer Architecture (ISCA'18). IEEE, 802-815.

[55] Omid Habibpour, Zhongxia Simon He, Wlodek Strupinski, Niklas Rorsman, and Herbert Zirath. 2017. Wafer scale millimeter-wave integrated circuits based on epitaxial graphene in high data rate communication. Sci. Rep. 7 (2017), 41828.

[56] Linden Harrison. 2006. An introduction to Depletion-mode MOSFETs. Retrieved from https://www.mikrocontroller. net/attachment/389314/IntroDepletionModeMOSFET.pdf.

[57] John L. Hennessy and David A. Patterson. 2011. Computer Architecture: A Quantitative Approach. Elsevier.

[58] John L. Hennessy and David A. Patterson. 2019. A new golden age for computer architecture. Commun. ACM 62, 2 (2019), 48-60.

[59] Gage Hills, Christian Lau, Andrew Wright, Samuel Fuller, Mindy D. Bishop, Tathagata Srimani, Pritpal Kanhaiya, Rebecca Ho, Aya Amer, Yosi Stein et al. 2019. Modern microprocessor built from complementary carbon nanotube transistors. Nature 572, 7771 (2019), 595-602.

[60] Jim Holmes, A. Matthew Francis, Ian Getreu, Matthew Barlow, Affan Abbasi, and H. Alan Mantooth. 2016. Extended high-temperature operation of silicon carbide CMOS circuits for Venus surface application. f. Microelectron. Electron. Packag. 13, 4 (2016), 143-154.

[61] Mark Horowitz, Elad Alon, Dinesh Patil, Samuel Naffziger, Rajesh Kumar, and Kerry Bernstein. 2005. Scaling, power, and the future of CMOS. In Proceedings of the IEEE International Electron Devices Meeting (IEDM'05). IEEE, 7-pp.

[62] Shuoben Hou. 2019. Silicon Carbide High-temperature Photodetectors and Image Sensor. Ph.D. Dissertation. KTH Royal Institute of Technology.

[63] Chenming Hu. 2010. Modern Semiconductor Devices for Integrated Circuits. Vol. 2. Prentice Hall, Upper Saddle River, NJ.

[64] Xing Hu, Dylan Stow, and Yuan Xie. 2018. Die stacking is happening. IEEE Micro 38, 1 (2018), 22-28.

[65] Yongjie Hu, Jie Xiang, Gengchiau Liang, Hao Yan, and Charles M. Lieber. 2008. Sub-100 nanometer channel length $\mathrm{Ge} / \mathrm{Si}$ nanowire transistors with potential for $2 \mathrm{THz}$ switching speed. Nano Lett. 8, 3 (2008), 925-930.

[66] Stephen Hudgens and Brian Johnson. 2004. Overview of phase-change chalcogenide nonvolatile memory technology. MRS Bull. 29, 11 (2004), 829-832.

[67] Clive Humby. 2006. Data is the new Oil. In Proceedings of the ANA Sr. Marketer's Summit.

[68] Gary W. Hunter, Philip G. Neudeck, Robert S. Okojie, Glenn M. Beheim, J. A. Powell, and Liangyu Chen. 2003. An overview of high-temperature electronics and sensor development at NASA glenn research center. F. Turbomach. 125, 4 (2003), 658-664.

[69] Rotem Ben Hur, Nimrod Wald, Nishil Talati, and Shahar Kvatinsky. 2017. SIMPLE MAGIC: Synthesis and inmemory mapping of logic execution for memristor-aided logic. In Proceedings of the 36th International Conference on Computer-Aided Design. IEEE Press, 225-232.

[70] IEEE. 2018. International Roadmap for Devices and Systems Beyond CMOS. Technical Report.

[71] IEEE. 2018. International Roadmap for Devices and Systems Moore Moore. Technical Report.

[72] Intel. 2019. Intel Core i9-9900K Processor. Retrieved from https:/www.intel.com/content/www/us/en/products/ processors/core/i9-processors/i9-9900k.html.

[73] Adrian M. Ionescu and Heike Riel. 2011. Tunnel field-effect transistors as energy-efficient electronic switches. Nature 479, 7373 (2011), 329.

[74] Ahmad Ehteshamul Islam. 2013. Variability and reliability of single-walled carbon nanotube field effect transistors. Electronics 2, 4 (2013), 332-367.

[75] Shubham Jain, Ashish Ranjan, Kaushik Roy, and Anand Raghunathan. 2017. Computing in Memory with Spintransfer Torque Magnetic Ram. IEEE Trans. Very Large Scale Integr. Syst. 26, 3 (2017), 470-483.

[76] Akhilesh Jaiswal and Kaushik Roy. 2016. Spin transfer torque memories for on-chip caches: Prospects and perspectives. In Proceedings of the 17th Latin-American Test Symposium (LATS'16). IEEE, 7-7.

[77] Raj Jana, Gregory L. Snider, and Debdeep Jena. 2013. Sub-Boltzmann transistors with piezoelectric gate barriers. In Proceedings of the 3rd Berkeley Symposium on Energy Efficient Electronic Systems (E3S'13). IEEE, 1-2.

[78] Matthew Jerry, Pai-Yu Chen, Jianchi Zhang, Pankaj Sharma, Kai Ni, Shimeng Yu, and Suman Datta. 2017. Ferroelectric FET analog synapse for acceleration of deep neural network training. In Proceedings of the IEEE International Electron Devices Meeting (IEDM'17). IEEE, 6-2.

[79] Javier Junquera and Philippe Ghosez. 2003. Critical thickness for ferroelectricity in perovskite ultrathin films. Nature 422, 6931 (2003), 506-509.

[80] Nam Sung Kim, Todd Austin, David Blaauw, Trevor Mudge, Jie S. Hu, Mary Jane Irwin, Mahmut Kandemir, Vijaykrishnan Narayanan et al. 2003. Leakage current: Moore’s law meets static power. Computer12 (2003), 68-75. 
[81] Daiki Kimoto. 2017. Characterization and Modeling of SiC Integrated Circuits for Harsh Environment. Ph.D. Dissertation. KTH Royal Institute of Technology.

[82] Kun Kong, Yun Shang, and Ruqian Lu. 2009. An optimized majority logic synthesis methodology for quantum-dot cellular automata. IEEE Trans. Nanotechnol. 9, 2 (2009), 170-183.

[83] Kunal Korgaonkar, Ishwar Bhati, Huichu Liu, Jayesh Gaur, Sasikanth Manipatruni, Sreenivas Subramoney, Tanay Karnik, Steven Swanson, Ian Young, and Hong Wang. 2018. Density tradeoffs of non-volatile memory as a replacement for SRAM-based last level cache. In Proceedings of the 45th Annual International Symposium on Computer Architecture. IEEE Press, 315-327.

[84] Zoran Krivokapic, U. Rana, R. Galatage, A. Razavieh, A. Aziz, J. Liu, J. Shi, H. J. Kim, R. Sporer, C. Serrao et al. 2017. $14 \mathrm{~nm}$ ferroelectric FinFET technology with steep subthreshold slope for ultra low power applications. In Proceedings of the IEEE International Electron Devices Meeting (IEDM'17). IEEE, 15-1.

[85] Emre Kultursay, Karthik Swaminathan, Vinay Saripalli, Vijaykrishnan Narayanan, Mahmut T. Kandemir, and Suman Datta. 2012. Performance enhancement under power constraints using heterogeneous CMOS-TFET multicores. In Proceedings of the 8th IEEE/ACM/IFIP International Conference on Hardware/Software Codesign and System Synthesis. ACM, 245-254.

[86] Shinichiro Kuroki, Hirofumi Nagatsuma, Milantha de Silva, Seiji Ishikawa, Tomonori Maeda, Hiroshi Sezaki, Takamaro Kikkawa, Takahiro Makino, Takeshi Ohshima, Mikael Östling et al. 2016. Characterization of 4H-SiC nMOSFETs in harsh environments, high-temperature and high gamma-ray radiation. In Materials Science Forum, Vol. 858. Trans Tech Publications, 864-867.

[87] Mark Lapedus. 2016. Mask maker worries grow. Retrieved November 3, 2019 from https://semiengineering.com/ what-mask-makers-want/.

[88] Benjamin C. Lee, Engin Ipek, Onur Mutlu, and Doug Burger. 2009. Architecting phase change memory as a scalable dram alternative. In Proceedings of the 36th Annual International Symposium on Computer Architecture. 2-13.

[89] Gyu-hyeon Lee, Dongmoon Min, Ilkwon Byun, and Jangwoo Kim. 2019. Cryogenic computer architecture modeling with memory-side case studies. In Proceedings of the 46th International Symposium on Computer Architecture. 774787.

[90] H. Y. Lee, Y. S. Chen, P. S. Chen, P. Y. Gu, Y. Y. Hsu, S. M. Wang, W. H. Liu, C. H. Tsai, S. S. Sheu, P. C. Chiang et al. 2010. Evidence and solution of over-RESET problem for HfO x-based resistive memory with sub-ns switching speed and high endurance. In Proceedings of the International Electron Devices Meeting. IEEE, 19-7.

[91] Yoonmyung Lee, Daeyeon Kim, Jin Cai, Isaac Lauer, Leland Chang, Steven J. Koester, David Blaauw, and Dennis Sylvester. 2013. Low-power circuit analysis and design based on heterojunction tunneling transistors (HETTs). IEEE Trans. Very Large Scale Integr. Syst. 21, 9 (2013), 1632-1643.

[92] David I. Lewin. 2002. DNA computing. Comput. Sci. Eng. 4, 3 (2002), 5-8.

[93] Jing Li, Binquan Luan, and Chung Lam. 2012. Resistance drift in phase change memory. In Proceedings of the IEEE International Reliability Physics Symposium (IRPS'12). IEEE, 6C-1.

[94] Shuangchen Li, Cong Xu, Qiaosha Zou, Jishen Zhao, Yu Lu, and Yuan Xie. 2016. Pinatubo: A processing-in-memory architecture for bulk bitwise operations in emerging non-volatile memories. In Proceedings of the 53rd Annual Design Automation Conference. ACM, 173.

[95] Xiao Li and Hongwei Zhu. 2015. Two-dimensional MoS2: Properties, preparation, and applications. F. Mater. 1, 1 (2015), 33-44.

[96] Yang Li, Kui Yao, and Ganesh Shankar Samudra. 2016. Effect of ferroelectric damping on dynamic characteristics of negative capacitance ferroelectric MOSFET. IEEE Trans. Electron Devices 63, 9 (2016), 3636-3641.

[97] Yu-Ming Lin, Joerg Appenzeller, Joachim Knoch, and Phaedon Avouris. 2005. High-performance carbon nanotube field-effect transistor with tunable polarities. IEEE Trans. Nanotechnol. 4, 5 (2005), 481-489.

[98] Tz-yi Liu, Tian Hong Yan, Roy Scheuerlein, Yingchang Chen, Jeffrey KoonYee Lee, Gopinath Balakrishnan, Gordon Yee, Henry Zhang, Alex Yap, Jingwen Ouyang et al. 2013. A $130.7 \mathrm{~mm}^{2}$ 2-layer 32-Gb ReRAM memory device in 24-nm technology. IEEE \}. Solid-State Circ. 49, 1 (2013), 140-153.

[99] Yongpan Liu, Fang Su, Yixiong Yang, Zhibo Wang, Yiqun Wang, Zewei Li, Xueqing Li, Ryuji Yoshimura, Takashi Naiki, Takashi Tsuwa et al. 2019. A 130-nm ferroelectric nonvolatile system-on-chip with direct peripheral restore architecture for transient computing system. IEEE J. Solid-State Circ. 54, 3 (2019), 885-895.

[100] Juan Pablo Llinas, Andrew Fairbrother, Gabriela Borin Barin, Wu Shi, Kyunghoon Lee, Shuang Wu, Byung Yong Choi, Rohit Braganza, Jordan Lear, Nicholas Kau et al. 2017. Short-channel field-effect transistors with 9-atom and 13-atom wide graphene nanoribbons. Nature Commun. 8, 1 (2017), 1-6.

[101] Gabriel H. Loh and Yuan Xie. 2010. 3D stacked microprocessor: Are we there yet?IEEE Micro 30, 3 (2010), $60-64$.

[102] Wei Lu and Charles M. Lieber. 2006. Semiconductor nanowires. J. Phys. D: Appl. Phys. 39, 21 (2006), R387. 
[103] Wei Lu, Jie Xiang, Brian P. Timko, Yue Wu, and Charles M. Lieber. 2005. One-dimensional hole gas in germanium/silicon nanowire heterostructures. Proc. Natl. Acad. Sci. U.S.A. 102, 29 (2005), 10046-10051.

[104] Paul Malfy. 2018. Intel Core i9-9900K processor review. Retrieved November 3, 2019 from https://www.moddersinc.com/intel-core-i9-9900k-processor-review/6/.

[105] Pravin Mane, Nishil Talati, Ameya Riswadkar, Ramesh Raghu, and C. K. Ramesha. 2015. Stateful-NOR-based reconfigurable architecture for logic implementation. Microelectron. f. 46, 6 (2015), 551-562.

[106] Thomas Mikolajick, Christine Dehm, Walter Hartner, Ivan Kasko, M. J. Kastner, Nicolas Nagel, Manfred Moert, and Carlos Mazure. 2001. FeRAM technology for high density applications. Microelectron. Reliabil. 41, 7 (2001), 947-950.

[107] T. Mikolajick, U. Schroeder, and S. Slesazeck. 2020. The past, the present, and the future of ferroelectric memories. IEEE Trans. Electron. Dev. 67, 4 (2020), 1434-1443.

[108] Dongmoon Min, Ilkwon Byun, Gyu-Hyeon Lee, Seongmin Na, and Jangwoo Kim. 2020. CryoCache: A fast, large, and cost-effective cache architecture for cryogenic computing. In Proceedings of the 25th International Conference on Architectural Support for Programming Languages and Operating Systems. 449-464.

[109] Umesh K. Mishra, Primit Parikh, Yi-Feng Wu et al. 2002. AlGaN/GaN HEMTs-an overview of device operation and applications. Proc. IEEE 90, 6 (2002), 1022-1031.

[110] Daniel Nagy, Guillermo Indalecio, Antonio J García-Loureiro, Muhammad A. Elmessary, Karol Kalna, and Natalia Seoane. 2018. FinFET versus gate-all-around nanowire FET: Performance, scaling, and variability. IEEE f. Electron Dev. Soc. 6 (2018), 332-340.

[111] Montassar Najari, Sebastien Fregonese, Cristell Maneux, Thomas Zimmer, Hassene Mnif, and N. Masmoudi. 2008. Towards compact modelling of Schottky barrier CNTFET. In Proceedings of the 3rd International Conference on Design and Technology of Integrated Systems in Nanoscale Era. IEEE, 1-6.

[112] Philip G. Neudeck, Liangyu Chen, Roger D. Meredith, Dorothy Lukco, David J. Spry, Leah M. Nakley, and Gary W. Hunter. 2018. Operational testing of $4 \mathrm{H}-\mathrm{SiC}$ JFET ICs for 60 days directly exposed to Venus surface atmospheric conditions. IEEE f. Electron. Dev. Soc. 7 (2018), 100-110.

[113] Philip G. Neudeck, Steven L. Garverick, David J. Spry, Liang-Yu Chen, Glenn M. Beheim, Michael J. Krasowski, and Mehran Mehregany. 2009. Extreme temperature 6H-SiC JFET integrated circuit technology. Physica Status Solidi (a) 206, 10 (2009), 2329-2345.

[114] Philip G. Neudeck, Roger D. Meredith, Liangyu Chen, David J. Spry, Leah M. Nakley, and Gary W. Hunter. 2016. Prolonged silicon carbide integrated circuit operation in Venus surface atmospheric conditions. AIP Adv. 6, 12 (2016), 125119.

[115] Philip G. Neudeck, Robert S. Okojie, and Liang-Yu Chen. 2002. High-temperature electronics-a role for wide bandgap semiconductors? Proc. IEEE 90, 6 (2002), 1065-1076.

[116] Philip G. Neudeck, David J. Spry, Liangyu Chen, Norman F. Prokop, and Michael J. Krasowski. 2017. Demonstration of $4 \mathrm{H}-\mathrm{SiC}$ digital integrated circuits above 800 C. IEEE Electron. Dev. Lett. 38, 8 (2017), 1082-1085.

[117] Philip G. Neudeck, David J. Spry, and Liang-Yu Chen. 2016. First-order SPICE modeling of extreme-temperature 4H-SiC JFET integrated circuits. Additional Papers and Presentations. HiTEC, 000263-000271.

[118] Kai Ni, Xunzhao Yin, Ann Franchesca Laguna, Siddharth Joshi, Stefan Dünkel, Martin Trentzsch, Johannes Müeller, Sven Beyer, Michael Niemier, Xiaobo Sharon Hu, et al. 2019. Ferroelectric ternary content-addressable memory for one-shot learning. Nature Electron. 2, 11 (2019), 521-529.

[119] Y. Nosho, Y. Ohno, S. Kishimoto, and T. Mizutani. 2006. Relation between conduction property and work function of contact metal in carbon nanotube field-effect transistors. Nanotechnol. 17, 14 (2006), 3412.

[120] Ugo Otuonye, Hee Woo Kim, and Wei D. Lu. 2017. Ge nanowire photodetector with high photoconductive gain epitaxially integrated on Si substrate. Appl. Phys. Lett. 110, 17 (2017), 173104.

[121] Hongsik Park, Ali Afzali, Shu-Jen Han, George S. Tulevski, Aaron D. Franklin, Jerry Tersoff, James B. Hannon, and Wilfried Haensch. 2012. High-density integration of carbon nanotubes via chemical self-assembly. Nature Nanotechnol. 7, 12 (2012), 787.

[122] Kyu-Sul Park, Sang-Jin Kim, In-Bok Baek, Won-Hee Lee, Jong-Seuk Kang, Yong-Bum Jo, Sang Don Lee, Chang-Keun Lee, Jung-Bum Choi, Jang-Han Kim et al. 2005. SOI single-electron transistor with low RC delay for logic cells and SET/FET hybrid ICs. IEEE Trans. Nanotechnol. 4, 2 (2005), 242-248.

[123] Eric Pop, David Mann, Qian Wang, Kenneth Goodson, and Hongjie Dai. 2006. Thermal conductance of an individual single-wall carbon nanotube above room temperature. Nano Lett. 6, 1 (2006), 96-100.

[124] Kiran Puttaswamy and Gabriel H. Loh. 2006. Thermal analysis of a 3D die-stacked high-performance microprocessor. In Proceedings of the 16th ACM Great Lakes Symposium on VLSI. ACM, 19-24.

[125] Moinuddin K. Qureshi, Vijayalakshmi Srinivasan, and Jude A. Rivers. 2009. Scalable high performance main memory system using phase-change memory technology. In Proceedings of the 36th Annual International Symposium on Computer Architecture. 24-33. 
[126] Branimir Radisavljevic, Aleksandra Radenovic, Jacopo Brivio, Valentina Giacometti, and Andras Kis. 2011. Singlelayer MoS 2 transistors. Nature Nanotechnol. 6, 3 (2011), 147.

[127] M. Radosavljević, St Heinze, J. Tersoff, and Ph Avouris. 2003. Drain voltage scaling in carbon nanotube transistors. Appl. Phys. Lett. 83, 12 (2003), 2435-2437.

[128] Nagarajan Raghavan, Daniel D. Frey, Michel Bosman, and Kin Leong Pey. 2015. Statistics of retention failure in the low resistance state for hafnium oxide RRAM using a Kinetic Monte Carlo approach. Microelectron. Reliabil. 55, 9-10 (2015), 1422-1426.

[129] D. Reinsel, J. Gantz, and J. Rydning. 2018. Data age 2025: The digitization of the world from edge to core. Retrieved from https://www. seagate. com/files/www-content/our-story/trends/files/idc-seagate-dataage-whitepaper. pdf.

[130] Bryan J. Rice, Vibhu Jindal, C. C. Lin, Jenah Harris-Jones, Harry Kwon, Andy Ma, Michael Goldstein, and Frank Goodwin. 2008. Overview of EUV Mask Metrology. Retrieved from https://www.nist.gov/system/files/documents/ pml/div683/conference/Rice_2011.pdf.

[131] Seyed Mohammad Seyedzadeh, Rakan Maddah, Alex Jones, and Rami Melhem. 2016. Leveraging ecc to mitigate read disturbance, false reads and write faults in STT-RAM. In Proceedings of the 46th Annual IEEE/IFIP International Conference on Dependable Systems and Networks (DSN'16). IEEE, 215-226.

[132] Ali Shafiee, Anirban Nag, Naveen Muralimanohar, Rajeev Balasubramonian, John Paul Strachan, Miao Hu, R. Stanley Williams, and Vivek Srikumar. 2016. ISAAC: A convolutional neural network accelerator with in situ analog arithmetic in crossbars. ACM SIGARCH Comput. Architect. News 44, 3 (2016), 14-26.

[133] Muhammad Shakir. 2019. Process Design Kit and High-temperature Digital ASICs in Silicon Carbide. Ph.D. Dissertation. KTH Royal Institute of Technology.

[134] Navin Shenoy. 2018. Intel's 2018 Data-Centric Innovation Summit. Retrieved October 22, 2019 from https://s21. q4cdn.com/600692695/files/doc_presentations/2018/08/2018_08_DCIS_Shenoy_Final.pdf.

[135] Max M. Shulaker, Gage Hills, Rebecca S. Park, Roger T. Howe, Krishna Saraswat, H.-S. Philip Wong, and Subhasish Mitra. 2017. Three-dimensional integration of nanotechnologies for computing and data storage on a single chip. Nature 547, 7661 (2017), 74.

[136] Max M. Shulaker, Gage Hills, Nishant Patil, Hai Wei, Hong-Yu Chen, H.-S. Philip Wong, and Subhasish Mitra. 2013. Carbon nanotube computer. Nature 501, 7468 (2013), 526.

[137] Benoît Sklénard, Philippe Blaise, Boubacar Traoré, Alberto Dragoni, Cécile Nail, and Elisa Vianello. 2017. Advances in the understanding of microscopic switching mechanisms in ReRAM devices. In Proceedings of the 47th European Solid-State Device Research Conference (ESSDERC'17). IEEE, 46-49.

[138] Young-Woo Son, Marvin L. Cohen, and Steven G. Louie. 2006. Energy gaps in graphene nanoribbons. Phys. Rev. Lett. 97, 21 (2006), 216803.

[139] Linghao Song, Xuehai Qian, Hai Li, and Yiran Chen. 2017. Pipelayer: A pipelined reram-based accelerator for deep learning. In Proceedings of the IEEE International Symposium on High Performance Computer Architecture (HPCA'17). IEEE, 541-552.

[140] Ed Sperling. 2016. Multi-patterning issues at 7nm, 5nm. Retrieved November 3, 2019 from https://semiengineering. com/multi-patterning-problems-grow/.

[141] David J. Spry, Philip G. Neudeck, Dorothy Lukco, Liang Yu Chen, Michael J. Krasowski, Norman F. Prokop, Carl W. Chang, and Glenn M. Beheim. 2018. Prolonged $500^{\circ} \mathrm{C}$ operation of $100+$ transistor silicon carbide integrated circuits. In Materials Science Forum, Vol. 924. Trans Tech Publications, 949-952.

[142] Y. Sugiyama, Y. Takeuchi, and M. Tacanot. 1992. Highly-sensitive InGaAs-2DEG Hall device made of pseudomorphic In0. 52A10. 48As/In0. 8Ga0. 2As heterostructure. Sensors Actuat. A: Phys. 34, 2 (1992), 131-136.

[143] Karthik Swaminathan, Emre Kultursay, Vinay Saripalli, Vijaykrishnan Narayanan, Mahmut T. Kandemir, and Suman Datta. 2013. Steep-slope devices: From dark to dim silicon. IEEE Micro 33, 5 (2013), 50-59.

[144] Nishil Talati, Ameer Haj Ali, Rotem Ben Hur, Nimrod Wald, Ronny Ronen, Pierre-Emmanuel Gaillardon, and Shahar Kvatinsky. 2018. Practical challenges in delivering the promises of real processing-in-memory machines. In Proceedings of the Design, Automation and Test in Europe Conference and Exhibition (DATE'18). IEEE, 1628-1633.

[145] Nishil Talati, Saransh Gupta, Pravin Mane, and Shahar Kvatinsky. 2016. Logic design within memristive memories using memristor-aided loGIC (MAGIC). IEEE Trans. Nanotechnol. 15, 4 (2016), 635-650.

[146] Nishil Talati, Heonjae Ha, Ben Perach, Ronny Ronen, and Shahar Kvatinsky. 2019. CONCEPT: A column-oriented memory controller for efficient memory and PIM operations in RRAM. IEEE Micro 39, 1 (2019), 33-43.

[147] Yuan Taur and Tak H. Ning. 2013. Fundamentals of Modern VLSI Devices. Cambridge University Press, Cambridge, UK.

[148] B. Turkot, S. Carson, and A. Lio. 2017. Continuing Moore's law with EUV lithography. In Proceedings of the IEEE International Electron Devices Meeting (IEDM'17. IEEE, 14-4.

[149] M. Vinet, P. Batude, C. Tabone, B. Previtali, C. LeRoyer, A. Pouydebasque, L. Clavelier, A. Valentian, O. Thomas, S. Michaud et al. 2011. 3D monolithic integration: Technological challenges and electrical results. Microelectron. Eng. 88, 4 (2011), 331-335. 
[150] Stefan Wachter, Dmitry K. Polyushkin, Ole Bethge, and Thomas Mueller. 2017. A microprocessor based on a twodimensional semiconductor. Nature Commun. 8 (2017), 14948.

[151] Han Wang, Lili Yu, Yi-Hsien Lee, Yumeng Shi, Allen Hsu, Matthew L. Chin, Lain-Jong Li, Madan Dubey, Jing Kong, and Tomas Palacios. 2012. Integrated circuits based on bilayer MoS2 transistors. Nano Lett. 12, 9 (2012), 4674-4680.

[152] Wikichip. 2019. Wikichip, 4004-Intel. Retrieved from https://en.wikichip.org/wiki/intel/mcs-4/4004.

[153] Wikipedia. 2019. Wikipedia, The Free Encyclopedia. Retrieved from https://en.wikipedia.org/wiki/Instructions_per_ second.

[154] Wikipedia. 2020. Wikipedia, The Free Encyclopedia. Retrieved from https://en.wikipedia.org/wiki/Ferroelectric_ RAM.

[155] Wikipedia. 2020. Wikipedia, The Free Encyclopedia. Retrieved from https://en.wikipedia.org/wiki/Fe_FET.

[156] H.-S. Philip Wong and Deji Akinwande. 2011. Carbon Nanotube and Graphene Device Physics. Cambridge University Press.

[157] H.-S. Philip Wong, Simone Raoux, SangBum Kim, Jiale Liang, John P. Reifenberg, Bipin Rajendran, Mehdi Asheghi, and Kenneth E. Goodson. 2010. Phase change memory. Proc. IEEE 98, 12 (2010), 2201-2227.

[158] Justin Wong and Sayeef Salahuddin. 2015. Piezoelectric Negative Capacitance. Ph.D. Dissertation. MA thesis. EECS Department, University of California, Berkeley.

[159] Jie Xiang, Wei Lu, Yongjie Hu, Yue Wu, Hao Yan, and Charles M. Lieber. 2006. Ge/Si nanowire heterostructures as high-performance field-effect transistors. Nature 441, 7092 (2006), 489.

[160] Cong Xu, Dimin Niu, Naveen Muralimanohar, Rajeev Balasubramonian, Tao Zhang, Shimeng Yu, and Yuan Xie. 2015. Overcoming the challenges of crossbar resistive memory architectures. In Proceedings of the IEEE 21st International Symposium on High Performance Computer Architecture (HPCA'15). IEEE, 476-488.

[161] B. Yang, K. D. Buddharaju, S. H. G. Teo, N. Singh, G. Q. Lo, and D. L. Kwong. 2008. Vertical silicon-nanowire formation and gate-all-around MOSFET. IEEE Electron. Device Lett. 29, 7 (2008), 791-794.

[162] Ran Yu, Yordan M. Georgiev, Samaresh Das, Richard G. Hobbs, Ian M. Povey, Nikolay Petkov, Maryam Shayesteh, Dan O'Connell, Justin D. Holmes, and Ray Duffy. 2014. Junctionless nanowire transistor fabricated with high mobility Ge channel. Rapid Res. Lett. 8, 1 (2014), 65-68.

[163] C.-M. Zetterling. 2013. Silicon carbide high-temperature electronics-Is this rocket science? Future Trends in Microelectronics: Frontiers and Innovations, Serge Luryi, Jimmy Xu, and Alex Zaslavsky (Eds.). John Wiley \& Sons, 102-109.

[164] Zhen Zhang and John T. Yates Jr. 2012. Band bending in semiconductors: Chemical and physical consequences at surfaces and interfaces. Chem. Rev. 112, 10 (2012), 5520-5551.

[165] Ping Zhou, Bo Zhao, Jun Yang, and Youtao Zhang. 2009. A durable and energy efficient main memory using phase change memory technology. ACM SIGARCH Comput. Architect. News 37, 3 (2009), 14-23.

[166] Mohammed A. Zidan, John Paul Strachan, and Wei D. Lu. 2018. The future of electronics based on memristive systems. Nature Electron. 1, 1 (2018), 22.

Received February 2020; revised October 2020; accepted January 2021 